\title{
The Role of Age in the Physiological Adaptations and Psychological Responses in Bikini-Physique Competitor Contest Preparation: A Case Series
}

Dan Newmire ( $\nabla$ daniel.newmire@tamucc.edu )

Texas A\&M University Corpus Christi https://orcid.org/0000-0001-8666-0728

Heather E. Webb

Texas A\&M University Corpus Christi

\section{Case report}

Keywords: Metabolism, Body composition, Skeletal muscle, Luteinizing hormone, Leptin

Posted Date: January 28th, 2021

DOI: https://doi.org/10.21203/rs.3.rs-152618/v1

License: (c) (i) This work is licensed under a Creative Commons Attribution 4.0 International License.

Read Full License

Version of Record: A version of this preprint was published at Journal of the International Society of Sports Nutrition on June 9th, 2021. See the published version at https://doi.org/10.1186/s12970-02100445-1. 


\section{Abstract}

Even with the increased popularity of the bikini-physique division within bodybuilding, there is minimal observational research identifying the influence of age on typical adaptations during contest preparation. The purpose of this case series was to observe how age may influence the adaptations normally seen during preparation and the exploration of newer protocols to address adaptations more relative to the judging standards. Over a 16-week pre-contest preparation, a 32-y bikini competitor (BC) and 44-y master's bikini competitor (MBC) visited the laboratory bi-weekly to observe changes in body fat mass $(B F)$, lean body mass (LBM), bone mineral density (BMD), total body water (TBW); exploratory measures of deltoid cross-sectional area (Delt $\left.t_{C S A}\right)$, gluteus maximus muscle thickness $\left(G_{M T}\right)$, and subcutaneous adipose tissue thickness (SAT); reproductive hormones estradiol (E2), luteinizing hormone (LH), and energy balance hormones triiodothyronine $\left(\mathrm{T}_{3}\right)$, leptin and ghrelin; hydration status during contest preparation and during the week of competition; resting metabolic rate (RMR); psychometric data related to perceived anxiety, stress, and body image were assessed. No differences between $B C$ and $M B C$ were observed in BF, LBM, BMD, and TBW. Both competitors showed a small loss in LBM. Both BC and MBC showed a contrasting increase in Delt ${ }_{\mathrm{CSA}}$ and a loss in $\mathrm{GM}_{\mathrm{MT}}$. $\mathrm{MBC}$ showed to be slightly more dehydrated (1.025 vs $1.021 \mathrm{~g} \cdot \mathrm{mL}^{-1}$ ) than $\mathrm{BC}$. Both competitors maintained a euhydration status the day of the competition. No time differences were found between $B C$ and MBC during RMR. BC showed a higher mean difference RMR compared to MBC $\left(2.66 \pm 0.75 \mathrm{kcal} \cdot \mathrm{kgLBM}^{-1} \cdot \mathrm{d}^{-1}\right)$. MBC showed a higher mean difference LH concentration $\left(84.6 \pm 6.01 \mathrm{IU} \cdot \mathrm{L}^{-1}\right)$, which may be explained by perimenopausal status. MBC had a higher mean difference concentration of leptin $\left(2.51 \pm 0.24 \mathrm{ng} \cdot \mathrm{mL}^{-1} \cdot \mathrm{kgFM}^{-1}\right)$, which was unperturbed by fat loss may be interrelated LH. BC self-reported a higher mean difference energy intake $\left(15.07 \pm 3.43 \mathrm{kcal} \cdot \mathrm{kgLBM}^{-1} \cdot \mathrm{d}^{-1}\right)$ and higher aerobic training volume $(93.26 \pm 40.68 \mathrm{~min} \cdot \mathrm{d})$. BC and MBC showed similar composition changes, slightly differing metabolic rates, and differing hormonal LH and leptin responses. This finding is in contrast to previous work showing both LH inhibition and leptin diurnal disturbance in younger, female athletes with low energy availability. The exploratory measures may have some benefit for bikini-physique competitors related to the judging criteria. Age did not seem to play a role in contest preparation adaptations.

\section{Introduction}

In 2010, the International Federation of Bodybuilding (IFBB) formally recognized bikini competitions as an independent competition category in the competitive physique category. Since its introduction, the bikini category has grown to become to be a popular division on the fitness stage and due to its' popularity, the bikini-physique category has expanded to include age-grouped competitions. Three agegroups currently exist, including the master's bikini-physique division (age $\geq 35 \mathrm{y}$ ), junior's level (age 16$23 \mathrm{y}$ ) and the remaining category for contestants between 23 and 34 years of age. According to the National Physique Committee (NPC; amateur) and IFBB (professional), female competitors in the bikini division are judged on these criteria: 1) muscular shape, "full roundness" of gluteus maximus; 2) a lower body fat composition to distinctly present segregated gluteal and hamstring muscle groups; 3) a "slight 
roundness" of the deltoid muscle group; 4) a very lean, low body fat abdominal region (http://npcnewsonline.com/bikini-rules/).

It has been shown that exercise training has a positive impact on the health of middle-aged women. As women age into their perimenopausal stage they experience a concurrent reduction in basal metabolic rate (BMR) and loss of lean body mass as they transition to menopause ${ }^{1}$. Changes in body composition (increased fat mass and decreased lean body mass) and in fat distribution (gynoid transition to android) seem to be influenced by the menopausal transition, as well as by chronological aging ${ }^{1}$. It he been shown that middle-aged women annually gain an average gain of $0.5 \mathrm{~kg}$ or more ${ }^{1}$. This weight gain and reduction of BMR is accompanied by reduced physical activity, as women significantly reduce regular exercise during middle age by $\sim 40 \%^{2}$. In contrast, active, middle-aged women tend to have an advantage as they enter the menopausal transition in terms of starting out with a lower BMI, lower fat mass, greater lean mass, decreased risk of obesity, higher associated increase in bone mineral density (BMD) in the femoral and spinal areas, and less android adiposity ${ }^{1}$. With the increased popularity of bikini-physique competitions and the known benefits for exercise training for middle-aged women, there is need to investigate how contest preparation may influence adaptations in middle-aged females. To our knowledge there are no current studies that have investigated and observed middle-aged female physique competitors to identify any notable impact age may have on contest preparation adaptations when compared to their younger cohort.

Recently, there has been a focus in observing differing categories of bikini-physique competitors during their competition preparation and any notable impact post-competition due limited the very limited data on this population ${ }^{3-11}$. Hulmi, et al. (2017) observed 50 competitors (27.2 \pm 4.1 y) over a $\sim 20$-week dieting phase followed by an 18-week recovery phase. Of the observed dieting phase group, 27 were IFBB amateur fitness competitors. Of these participants, 17 were bikini-physique competitors ${ }^{12}$. They found that the decreased energy intake in the diet group was mainly explained by the reduction of carbohydrate $(\mathrm{CHO})$ intake with only very slight decreases in fat and no changes in protein intake were noticed. They observed that BF\% decreased from $23.1 \pm 5.6$ to $12.7 \pm 4.0 \%$ measured via DXA. Neither the diet nor recovery phase had much impact on changes in lean body mass (LBM) seen in the dieting group assessed by DXA. However, they did see a small decrease in ultrasound (US) assessed vastus lateralis CSA due to the diet phase. Hormone concentrations of leptin, testosterone, and triiodothyronine $\left(T_{3}\right)$ reduced during the dieting phase ${ }^{12}$.

Mathison, et al. (2019) observed a categorically heterogenous population of female physique competitors $(28.1 \pm 5.5 \mathrm{y})$. Of the 25 subjects competing, 21 were categorized as bikini-physique competitors ( 4 were defined as "body and fitness athletic" categories). The subjects were assessed three times during their contest preparation and compared to a similar non-contest preparation group of female physique competitors. The subjects were assessed at baseline, 2-weeks pre-competition, and 1-month postcompetition. They assessed body composition via dual-energy $x$-ray absorptiometry (DXA) scan, resting metabolic rate (RMR) via indirect calorimetry, 4-day dietary recall and physical activity questionnaire, and 
psychometric data. They found that competitors that did not use hormone contraceptives had greater menstrual irregularity than those that reported use. Dietary analysis showed that both groups $\mathrm{CHO}$ intake were below recommendations $\left(5-7 \mathrm{~g} \cdot \mathrm{kg}^{-1} \cdot \mathrm{d}^{-1}\right)^{13}$. Resting metabolic rate (RMR) was suggested to be clinically low based on their comparative reference using Cunningham Eq. $1^{4}$, which may suggest an energy deficiency. The contest preparation group showed the lowest RMR 2-weeks prior to their contest. Interestingly, the contest preparation group showed a slightly higher value of LBM at 2-weeks prior to competition time point with a significantly lower reported Kcal intake $\left(33 \mathrm{~g} \cdot \mathrm{kgLBM}^{-1} \cdot \mathrm{d}^{-1}\right)$. The authors summarized that due to the low (kilocalorie) kcal intake reported $\left(33 \mathrm{~g} \cdot \mathrm{kgLBM}^{-1} \cdot \mathrm{d}^{-1}\right)$ at 2-weeks prior to their contest was categorized as low energy availability (LEA) due to this value being uncorrected controlling for exercise energy expenditure from exercise training as previously recommended equation Energy Availability $(E A)=$ Energy Intake $(E I)-$ Exercise Energy Expenditure $(E E E)^{15}$. The recommended threshold for maintenance for female athletes to maintain normal eumenorrhea is $30 \mathrm{~g} \cdot \mathrm{kgLBM}^{-1} \cdot \mathrm{d}^{-1}$, the authors suggested that this may explain some of the changes they observed ${ }^{16}$.

Longstrom, et al. (2020) recently published a post-contest focused outcome case series observing a sample population of four female-physique competitors (age $29.3 \pm 4.9 \mathrm{y}$ ), that included two figure- and two bikini-physique competitors. Thankfully, in their analysis they separated individual subject findings. They collected data at three time points: 1-2 weeks pre-competition, four weeks and 8-10 weeks postcompetition. They assessed body composition using skinfold technique, total body water using multifrequency bioelectrical impedance analysis (MF-BIA), RMR, hormonal responses, muscular endurance, nutritional analysis, and subjective psychometric data that assessed sleep habits, quality of life, and menstrual cycle. They compared the change from the time point of 1-2 weeks prior to competition to 8-10 weeks post-competition. Focusing on bikini-physique competitors and between the time points of 1-2 weeks prior to competition and 8-10 weeks post-competition, they found that the adipokine hormone leptin that is synthesized from white adipose tissue, increased relative to the increase in fat mass gain. They also showed that the increase in $\mathrm{kg}$ fat mass increased total $\mathrm{kg}$ body weight and therefore were directly related to RMR. Lastly, when highlighting other hormonal changes, both $\mathrm{T}_{3}$ and thyroxine $\left(T_{4}\right)$ slightly increased from pre-competition measures.

As difficult as it is to accurately assess, report, and control in these observational studies, each of these previous investigations mentioned previously highlighted specific questions related to female-physique competitors. However, due the inherent analysis of a heterogenic population of differing categories of female competitors, limited sample collection time periods, and a comparing pre-competition status with post-competition status, it is difficult discern more specific contest preparation responses to reference and compare for bikini-physique competitors. Each female-physique category will have differing judging criteria, which may dictate the selected dieting and training protocols, and therefore the physiological and psychological responses. Additionally, some of the skeletal muscle measures used to assess the femalephysique population ${ }^{17}$ in these previous investigations have been well validated yet may lack an ability to generalize towards bikini-physique competitors relative to their judging criteria. Lastly, to our knowledge there are currently no observational studies investigating the impact of contest preparation on masters- 
female competitors. The investigations mentioned previously recruited a demographical focused age range of $\sim 23-34 \mathrm{y}$. The purpose of this case series was to observe, follow, and analyze physiological

and psychological measures in two differing age categories in female bikini-physique competitors preparing for a professional qualifying, national competition. In comparison to previous studies, our focus was observational time course study during the contest preparation phase while comparing any notable physiological and psychological differences between age. Additionally, our goal was to collect novel and exploratory measures that could be useful for this population based on the judging criteria for these contests.

\section{Materials And Methods}

\section{Participants and Ethical Approval}

Two female bikini-physique participants (bikini competitor [BC]; master's bikini competitor [MBC]) were recruited during this study. Unfortunately, due to the COVID-19 pandemic, numerous local, regional, and national contests during the Summer and Fall 2020 NPC competitive season were canceled, postponed, and/or relocated, thus reducing our ability to recruit and retain participants. The participants' baseline characteristics are located below in Table 1. Both participants self-reported a regular menstruation cycle throughout the contest preparation. Additionally, both reported not using any form of birth control or pharmacological ergogenic aids. Supplement use was reported to be multivitamins, carnitine, and creatine for the BC and Vitamin E, Biotin, Zinc, Collagen, and Iron for MBC. This study was approved by the Texas A\&M University-Corpus Christi Institutional Review Board (\#TAMU-CC-IRB-2020-02-027), and the Institutional Biosafety Committee. 
Table 1

\begin{tabular}{|c|c|c|}
\hline Participant Characteristics & BC & MBC \\
\hline Age (y) & 32 & 44 \\
\hline Height (cm) & 163.2 & 156.2 \\
\hline Weight (kg) & 55.9 & 53.07 \\
\hline Body Fat (\%) & 21.8 & 19.5 \\
\hline Body Fat Mass (kg) & 11.71 & 9.98 \\
\hline Lean Body Mass (kg) & 42.14 & 41.27 \\
\hline Skeletal Muscle Mass (kg) & 21.22 & 19 \\
\hline Bone Mineral Density $\left(\mathrm{g} \cdot \mathrm{cm}^{-2}\right)$ & 1.119 & 1.101 \\
\hline BMD Age Matched Z-Score (\%) & 106 & 105 \\
\hline RT Frequency $\left(\mathrm{d} \cdot\right.$ week $\left.^{-1}\right)$ * & $4-6$ & $5-6$ \\
\hline AT Frequency $\left(\mathrm{d} \cdot\right.$ week $\left.^{-1}\right)$ * & 6 & 6 \\
\hline Competition Experience $(y)$ * & 3 & 2 \\
\hline
\end{tabular}

\section{Experimental Observational Design}

Testing procedures occurred bi-weekly during the participants 16-week pre-contest preparation are shown in Table 2. Following the competition (week 16 to 20), the BC elected to end contest preparation to begin a reverse dieting protocol and lower the volume of exercise training. The MBC elected to continue contest preparation with the goal of competing in a subsequent competition (competition to week 20). The participants visited the laboratory at 0800 every session after fasting for 8-10 h. Participants were instructed to not eat or drink prior to assessment. Monthly procedures consisted of body composition via Dual X-ray Absorptiometry (iDXA) for body fat mass (FM), body fat \% (BF\%), lean body mass (LBM), and bone mineral content (BMC); ultrasound (US) for subcutaneous adipose tissue (SAT) thickness, and exploratory measures of deltoid cross-sectional area (Delt $\mathrm{CSA}_{\mathrm{A}}$ ) and gluteus maximus muscle thickness $\left(G_{M T}\right)$, and total body water (TBW) was assessed with multifrequency bioelectrical impedance analysis (MF-BIA). Urine samples were collected upon their arrival for hydration status using urine specific gravity USG) analysis. Venous blood sampling for hormone analysis took place post-RMR to minimize any impact of stress on metabolic rate measures. Finally, psychometric information using the Perceived Stress Scale; (PSS) ${ }^{18}$, Body Image States Scale (BISS) ${ }^{19}$, and dietary and exercise training recall data were also collected. Bi-weekly procedures included MF-BIA, hydration status via USG, psychometrics, and dietary and exercise training recall. Further, baseline and pre-competition values of body image and 
eating behaviors were collected using the Body Appreciation Scale (BAS-2) ${ }^{20}$, Social Physique Anxiety Scale (SPAS) ${ }^{21}$, and the Eating Attitudes Test 26 (EAT-26) ${ }^{22}$. Due to the effect of COVID-19 on gym closures, the contest preparation start date was adapted. The baseline values for both participants were 16-weeks from the competition they had originally planned.

\begin{tabular}{|c|c|c|c|c|c|c|c|c|c|c|c|c|}
\hline & \multicolumn{12}{|l|}{ Table 2} \\
\hline & \multirow[t]{2}{*}{ Measure } & \multicolumn{11}{|l|}{ Weeks } \\
\hline & & Baseline (1) & 2 & 4 & 6 & 8 & 10 & 12 & 14 & 16 & 18 & 20 \\
\hline 1 & iDXA & $x$ & & $\mathrm{x}$ & & $x$ & & $x$ & & $x$ & & $x$ \\
\hline 2 & MF-BIA & $x$ & 0 & $\mathrm{x}$ & 0 & $x$ & 0 & $x$ & 0 & $x$ & 0 & $x$ \\
\hline 3 & US & $x$ & & $x$ & & $x$ & & $X$ & & $x$ & & $x$ \\
\hline 4 & Blood Sample & $x$ & & $\mathrm{x}$ & & $x$ & & $x$ & & $X$ & & $X$ \\
\hline 5 & Urine Sample & $x$ & 0 & $x$ & 0 & $x$ & 0 & $x$ & 0 & $x$ & 0 & $x$ \\
\hline 6 & RMR & $x$ & & $x$ & & $x$ & & $x$ & & $x$ & & $x$ \\
\hline 7 & PSS \& BISS & $x$ & 0 & $x$ & 0 & $x$ & 0 & $x$ & 0 & $x$ & 0 & $x$ \\
\hline 8 & BAS-2, EAT-26, \& SPAS & Z & & & & & & & & Z & & \\
\hline 9 & Dietary Recall & $x$ & 0 & $x$ & 0 & $x$ & 0 & $x$ & 0 & $x$ & 0 & $x$ \\
\hline \multirow[t]{2}{*}{10} & Exercise Training Recall & $x$ & 0 & $x$ & 0 & $x$ & 0 & $x$ & 0 & $x$ & 0 & $x$ \\
\hline & \multicolumn{12}{|c|}{$\begin{array}{l}\text { Dual X-ray Absorptiometry (iDXA); Multi-frequency bioelectrical impedance analysis (MF-BIA); } \\
\text { Ultrasound (US); Resting metabolic rate (RMR); Perceived Stress Scale (PSS); Body Image } \\
\text { States Scale (BISS); Body Appreciation Scale (BAS-2); Eating Attitudes Test-26 (EAT-26); Social } \\
\text { Physique Anxiety Scale (SPAS); X = Monthly data collection; O = Bi-weekly data; Z = Baseline and } \\
\text { pre-contest collection; Dashed red line = competition. }\end{array}$} \\
\hline
\end{tabular}

\section{Body Composition Analysis}

The participants height and weight were collected prior to compositional measures using a digital scale and stadiometer (SECA 769; Chino, CA). Body composition was assessed with iDXA (iDXA, Lunar Prodigy; GE Healthcare, Madison, WI) following International Society for Clinical Densitometry protocol recommendations ${ }^{23}$ to capture $\mathrm{FM}, \mathrm{BF} \%, \mathrm{LBM}$, and BMC. Further, using a previous equation shown to be a reliable and accurate estimation of skeletal muscle mass (SKMM) by Kim, et al. (20020), we estimated SKMM from appendicular soft lean tissue (ASLT) ${ }^{24}$. To assess changes in body water, we utilized InBody 720 (InBody USA; Cerritos, CA). It has been previously shown that MF-BIA analysis has a $\mathrm{R}^{2}(0.82-0.86)$ and SEE (1.5-1.6 kg) in adult females when compared to the gold standard of total body water measure, isotopic deuterium dilution $\left(\mathrm{D}_{2} \mathrm{O}\right)^{25}$. Total body water was assessed bi-weekly and conveniently upon the return of the competitors 5 -days post-competition to observe any notable fluid changes from post- 
competition hyperphagia similar to the state of weight recovery induced by post-starvation hyperphagia found in previous energy restriction studies ${ }^{26}$.

Roughly, $80-90 \%$ of fat mass is stored subcutaneously ${ }^{27}$, to address local subcutaneous adipose tissue (SAT) changes directly, we utilized the US (GE Logiq E9 (GE Healthcare, Wauwatosa, WI) using B-mode system with a wide band linear array transducer (L4-12t-RS) operating between $4.2-13 \mathrm{MHz}$ with $12.7 \mathrm{x}$ $47.1 \mathrm{~mm}$ footprint. Following ACSM protocol recommendations ${ }^{28}$, the 7-site areas of the right side of the body were quantified following a previously published procedure ${ }^{5}$. The measurement sites included the chest, triceps, subscapular, midaxillary, suprailiac, abdomen, and anterior thigh, which were located using standard anatomical landmarks. The linear transducer was coated in water-soluble transmission gel which enabled acoustic contact without depression of the skin and SAT. The transducer was maintained perpendicular and two images were taken per site and SAT thickness was measured from the skin to the inside edge of the superficial fascia using NIH ImageJ software (http://imagej.nih.gov/ij/). Each image was captured by the same investigator. Previous literature has validated $(I C C=.99)$ using US for SAT analysis compared to $\mathrm{MRI}^{29}$ and B-mode US has been validated to accurately assess SAT in previous study ${ }^{30}$. Furthermore, the 7-site measures were used to estimate BF\% using the appropriate equation to compare to iDXA ${ }^{31}$. The inter-SAT CV\% measure was $4.28 \%$

\section{Exploratory Regional Skeletal Muscle Analysis}

The use of US to quantify muscular adaptations of both CSA and MT in the vastus lateralis has been validated to $\mathrm{MR}^{32-35}$. However, the muscular adaptations in the regional area of the quadricep may be of less interest in the sample population we selected to observe. Based on the judging criteria listed above, both the muscular adaptations in the deltoid and gluteus maximus muscle groups may be of more importance and relative to meet bikini-physique judging requirements. Utilizing the same US unit, we acquired a panoramic LOGIQView ${ }^{\circledR}$ or extended field of view (EFOV) of the deltoid to assess crosssectional area changes (Delt $\mathrm{CSA}_{\mathrm{A}}$ ). Due to the novelty of and exploratory nature of this measure, we collected 2 scans of the largest semi-circumference of each competitor's right arm, deltoid area (Fig. 1). The participant was instructed to sit on examination table with arm relaxed at their side. Two lines were drawn to follow a path for the transducer to follow. The transducer was coated with copious amount of water-soluble transmission gel and maintained perpendicular to skin during with minimal depression of the skin. Two images were taken at each session. Due to the complexity of the muscular anatomy segments of the deltoid and quality of the US image, we were unable to discern definitive aponeuroses among anterior (A1, A2, A3) medial (M1), and posterior deltoid (P1, P2, P3) on all images. Due to this we elected to quantify the deltoid CSA cumulatively. The Delt ${ }_{\text {CSA }}$ was analyzed using NIH ImageJ. The same investigator acquired all the images of the Delt $\mathrm{CSA}_{\text {. The inter-Delt }}$ CSA $\mathrm{CV} \%$ measure was .53\%.

The image on the left portrays the area of interest where the largest semi-circumference of the right deltoid was located and marked. Two lines were made above and below the tape measure. The transducer followed a path from the pectoralis major and the anterior deltoid interact to the furthest point posterior to capture the most medial portion of the posterior deltoid. The image on the right is the Delt $\mathrm{CSA}_{\text {. }}$ 
Additionally, we wanted to explore gluteus maximus ( $\left.\mathrm{GM}_{\mathrm{MT}}\right)$ adaptations during their contest preparation as this measure may hold a referring value to bikini-physique competitors and the contest judging requirements. Using a similar protocol in published study exploring GM assessment with US36, the participant was asked to lay prone and legs comfortably adducted. To determine the transducer placement, the examiners palpated from the participant's posterior superior iliac spine (PSIS) to the ischial tuberosity (IT). After the IT was found, the participant was instructed to pull up their clothing, so their skin was available to again be palpated and the IT position was marked with a surgical pen (Fig. 2). Using B-mode imaging, the IT was found using a depth of $12 \mathrm{~cm}$ and two transverse images were taken by the same investigator. The $\mathrm{GM}_{\mathrm{MT}}$ was measured in a straight line from the superficial aponeurosis of the $\mathrm{GM}_{\mathrm{MT}}$ muscle, extending to the deep aponeurosis near the IT. The inter-GM $\mathrm{MT}_{\mathrm{MT}} \mathrm{CV} \%$ measure was .48 $\%$.

The image of the left shows the marked location of the ischial tuberosity (IT). The image on the right shows the US measurement taken to assess $\mathrm{GM}_{\mathrm{MT}}$.

\section{Hydration Status}

Participants were asked to provide a urine sample after arrival to the laboratory or maintained in a cryogenic status during competition and transport back to laboratory. Additionally, each participant traveled to their competition with urine collection cups to observe competition week and competition hydration status. The participants were instructed to collect samples upon waking and place the sealed sample in sealed plastic bag, and then store the sample in the freezer portion of a mid-sized refrigerator/freezer in their hotel suite. The 5-day competition week samples were then cryo-shipped in an insulated container back to laboratory. Hydration status was assessed by urine specific gravity (USG) by aspirating 1-3 drops of the urine sample onto the lens of a digital scale clinical refractometer (Sper Scientific; Model 300005; Scottsdale, AZ). The accuracy of the clinical refractometer is between \pm 0.002 and the refractive index changes proportionally to urine concentrations. Prior to urine USG analysis, both doubly distilled de-ionized water $\left(\mathrm{DDH}_{2} \mathrm{O}\right)$ and a prepared known density $\left(1.020 \mathrm{~g} \cdot \mathrm{mL}^{-1}\right)$ of $\mathrm{NaCl}$ and $\mathrm{DDH}_{2} \mathrm{O}$ were analyzed in duplicate for reference values. Urine samples were analyzed in duplicate using standard procedures. Urinary USG sample values were then compared to the validated index of associated hydration status ${ }^{37}$ values listed below in Table 3. 


\section{Table 3}

Index for Hydration Status

\begin{tabular}{|lc|}
\hline Condition & USG Value \\
\hline Well hydrated & $<1.010$ \\
\hline Minimal dehydration & $<1.010-1.020$ \\
\hline Significant dehydration & $1.021-1.030$ \\
\hline Serious dehydration & $>1.030$ \\
\hline Urine Specific Gravity (USG); $\leq 1.020$ is an indication of euhydration status & 38 \\
\hline
\end{tabular}

\section{Resting Metabolic Rate}

Resting metabolic rate (RMR) was assessed with indirect calorimetry (TrueOne 2400 Canopy System, ParvoMedics, Sandy, UT, USA). The TrueOne 2400 dilution mode system was calibrated prior to each assessment following manufacturer recommendations. Participants were asked to relax and lie supine on an examination table with the head rest set at an incline of $\sim 45^{\circ}$ during the 22-min assessment period and to maintain alertness with eyes open. The canopy was then placed over the head, shoulders, and upper chest of the participant to reduce environmental air to contaminating sample air entering or expired air escaping during measurement. The flow rate was established at $\sim 28$ to $30 \mathrm{ml} \cdot \mathrm{min}^{-1}$ within the first 1$3 \mathrm{~min}$ of the assessment as per manufacturer's instructions. The flow rate was adjusted to maintain the diluted $\mathrm{CO}_{2}$ percentage at $\sim 1 \%$ during testing. The first $10 \mathrm{~min}$ of the testing procedure were discarded, and the following 12-min of the test were averaged. The percent between the measured RMR (RMR $\left.{ }_{\text {Meas }}\right)$ and the estimated RMR (RMR $\left.\mathrm{R}_{\text {Calc }}\right)$ using the LBM Cunningham formula ${ }^{14}$. A RMR $\mathrm{Reas}_{\text {Sea }} / \mathrm{RMR}_{\text {Calc }} \%$ between $90-110 \%$ is considered normal and is commonly used as a threshold for diagnosis of clinically low RMR, indicating of energy deficiency ${ }^{11,39}$. Measured RMR will be expressed in both $\mathrm{Kcal}^{-\mathrm{d}^{-1}}$ and $\mathrm{Kcal} \cdot \mathrm{kgLBM}^{-}$ ${ }^{1} \cdot d^{-1}$ due to organ tissue being more metabolically active than skeletal muscle tissue in resting conditions, and LBM factor that explains greater proportion of the variability in $\mathrm{RMR}^{40}$.

\section{Dietary and Exercise Training Recall}

Participants followed guidance from a contest preparation coach. self-reported bi-weekly dietary macronutrient intake data was collected using MyfitnessPal. Hand held smart phone dietary tracking apps such as MyFitnessPal have shown to be more practical and a relative validity in comparison to other dietary recall procedures ${ }^{41}$. For exercise training recall, the investigators designed a training recall to collect data related to resistance training (RT) frequency and exercise selection. Additionally, participants reported sets, repetitions, and weight (Ibs) to quantify training load volume (sets.reps $\cdot \mathrm{kg}$ ) during contest preparation ${ }^{42,43}$. For aerobic training (AT) recall, participants reported time, frequency, exercise selection, duration, and RPE of the bout, which will be expressed in min.d. Due to the inherent difficulty of capturing 
accurate self-reported intensity of AT bouts, we were unable accurately estimate aerobic energy expenditure and how that may influence energy availability.

\section{Blood Sampling and Biochemistry Analysis}

A $4 \mathrm{~mL}$ blood samples were collected in $\mathrm{K}_{2}$ EDTA tubes that were acquired by a certified phlebotomist following WHO guidelines ${ }^{44}$. Samples were subsequently centrifuged at $3000 \mathrm{rpm}$ at $4^{\circ} \mathrm{C}$ for $10 \mathrm{~min}$.

Plasma samples $(500 \mathrm{~mL})$ were then transferred into storage microcentrifuge tubes and frozen at $-80^{\circ} \mathrm{C}$ until later analysis. A small blood sample was used for hematocrit testing to determine any notable changes in plasma volume (PV\%). Hormone analysis was completed utilizing readily available ELISA kits that included Estradiol (MBS2606149), Luteinizing Hormone (LH) (MBS047228), total T 3 (MBS580156), leptin (MBS020274), and total ghrelin (MBS3804142). All hormones were expressed in the units supplied by manufacturer. However, due to the hormone leptin being primarily synthesized by adipose tissue, leptin is also expressed per kg of FM. Intra-assay CV\% concentration was found to be $6.82 \%$. The mean PV\% for each competitor was $47.12 \pm 1.33 \%$. It is to be noted that an early study hematocrit \% analysis showed a competitor had very low value and they were suggested to talk to their physician to make adjustments to increase their hematocrit to normal levels. No other issues were seen in hematocrit moving forward.

\section{Psychometrics}

To explore the potential influence of self-perceived body image, stress, and eating behaviors on exercise behaviors, psychometric measurements were also collected, utilizing a computer-based survey system to collect this data. Baseline and pre-competition values of body image and eating behaviors were collected using the Body Appreciation Scale (BAS-2) ${ }^{20}$, Social Physique Anxiety Scale (SPAS) ${ }^{21}$, and the Eating Attitudes Test-26 (EAT-26) ${ }^{22}$, while the Perceived Stress Scale (PSS) ${ }^{18}$ and Body Image States Scale (BISS) ${ }^{19}$ were collected during each bi-weekly data collection session. The BAS-2 was developed to assist in evaluating an individual's perception of self-image. The BAS-2 is a ten-question, Likert-type scale on which statements are rated on a 5-point scale ranging from Never (receiving a score of 1) to Always (receiving a score of 5). The BAS-2 has valid psychometric properties, with previous studies reporting very good Cronbach's a internal consistency values ranging from .87 to .93 in women ${ }^{20}$.

Hart, et al. (1989) devised the SPAS to measures social anxiety related to an individual's physique, specifically the body's form and structure with a focus on body fat, muscular tone, and general body proportions, which mimic the desirable characteristics in the bikini-physique competition category. The SPAS is a 12-item self-report scale developed to assess the degree to which people become anxious when others observe or evaluate their physiques, on a scale of 1 (never) to 5 (always). The SPAS has high internal and test-retest reliability, with a Cronbach's alpha coefficient of .90 and an eight-week test-retest reliability coefficient of $.80^{21}$.

The BISS is a six-item measure of individuals' evaluation and affect about their physical appearance at a particular moment in time. Participants respond to six prompts on a 9-point, bipolar, Likert-type scales regarding satisfaction-dissatisfaction with their overall physical appearance, body size and shape, their 
weight, feelings of attractive or unattractiveness, current feelings of how they look compared to how they feel, and their own appearance relative to the average person ${ }^{19}$.

The PSS was asked bi-weekly to evaluate the extent of stress and lack of control that each participant had felt during that time period. The PSS is a 10-item inventory scored on a Likert-type scale from 0 (never) to 4 (very often) about feelings and thoughts during the preceding month. Values on the PSS can range from 0 to 40, with increased values representative of greater perceptions of stress. Coefficient alpha reliability for the PSS was shown to be $0.84,0.85$, and 0.86 in three separate samples, with a testretest correlation of $0.85^{18}$.

The EAT-26 22 (Garner, 1982) is a self-report questionnaire with 26-items that measures symptoms and concerns characteristic of eating disorders. The EAT-26 is scored using a six-point scale based on how often the individual engages in specific behaviors, ranging from always to never. Although the EAT-26 will yield a "referral index" based on three criteria, only the total score based on the answers to the EAT-26 questions were utilized in this study. Test-retest reliability for the EAT-26 ranges from 0.84 to $0.89^{45}$.

\section{Statistical Analysis}

Due to the inherent nature of this observational case series, the time course data will be expressed in a descriptive in nature to highlight any notable differences the impact age may have on the adaptations seen during contest preparation in bikini-physique competitors. The observational and exploratory nature of this study and the outcomes highlighted may be used to assist in driving future studies with larger sample groups, rather than forming any definitive conclusions and relationships. Tables are presented changes from Baseline (1) to 16-weeks (pre-competition) was calculated in tables for unit change $(\triangle)$ and percent change $(\triangle \%)$. Recommended or normal ranges were provided when appropriate for comparison from the ABIM Laboratory Test Reference Ranges and other qualifying organizations ${ }^{13}, 38,39$, 46. Additionally, both 4-day dietary recall and the exploratory analysis of RMR data were assessed with a 2-way ANOVA (time $x$ competitor) using Geisser-Greenhouse correction and Sidak post hoc analysis. Further, dietary recall data was averaged and expressed in $\mathrm{kcal} \cdot \mathrm{kg}^{-1} \cdot \mathrm{d}^{-1}$ and $\mathrm{kcal} \cdot \mathrm{kgLBM}^{-1} \cdot \mathrm{d}^{-1}$ to compare to recommended values. The mean average of resistance training volume load and aerobic training were compared between participants. The macronutrients were further expressed in $\mathrm{g} \cdot \mathrm{kg}^{-1} \cdot \mathrm{d}^{-1}$ to compare to recommended intake ranges. Typically, RMR is expressed $\left(\mathrm{kcal} \cdot \mathrm{d}^{-1}\right)$ and compared between participants or to baseline. However, due the variance of LBM on RMR measures ${ }^{40}$, and skeletal muscle metabolism being a major determinant of $\mathrm{RMR}^{47}$, data was normalized per $\mathrm{kg}$ of LBM to more fairly evaluate any differences. Similarly, the hormone leptin was expressed per kg FM. When appropriate, unpaired parametric student $t$-tests were used to compare mean differences between $\mathrm{BC}$ and $\mathrm{MBC}$ following Shapiro-Wilk test of normality. If normality testing failed, Welch's correction was used. Data will be expressed in mean \pm SEM along with $95 \%$ confidence intervals $(95 \% \mathrm{Cl})$ where appropriate. Pearson's $r$ correlation coefficient was used when appropriate to highlight any potential relationships that assist in explaining outcomes and further generate future questions related to female-physique competitor studies. The statistical power (1- $\beta$ error probability) was set at 0.8 , a error probability at $p=0.05$. All 
statistical analysis and figure construction were done using GraphPad PRISM software (version 9.0; GraphPad Software Inc., San Diego, CA, USA).

\section{Results}

Body Composition

Figure 3: The time course analysis of 20-week contest preparation of A) Weight, B) Fat mass, C) Total subcutaneous adipose tissue (SAT), and D) Body fat \%. Each time course analysis was accompanied with 4-week interval $\triangle$ change (mean \pm SEM). No significant differences were found in mean change between $\mathrm{BC}$ and $\mathrm{MBC}$. The dashed red line denotes the competition.

Figure 4: The time course analysis of 20-week contest preparation of $\mathbf{A}$ ) lean body mass (LBM) and $\mathbf{B}$ ) estimated skeletal muscle mass (SKMM). Each time course analysis was accompanied with 4-week interval $\triangle$ change (mean \pm SEM). No significant differences were found in mean change between $B C$ and MBC. The dashed red line denotes the competition.

Figure 5: The time course analysis of 20-week contest preparation of A) total body water (TBW), B) intracellular fluid (ICF), and C) extracellular fluid (ECF). Each time course analysis was accompanied with bi-weekly interval $\triangle$ change (mean \pm SEM). No significant differences were found in mean change between $\mathrm{BC}$ and $\mathrm{MBC}$. The dashed red line denotes the competition.

Figure 6: The time course analysis of 20-week contest preparation of $\mathbf{A}$ ) bone mineral content (BMC) and B) bone mineral density (BMD). Each time course analysis was accompanied with 4-week interval $\Delta$ change (mean \pm SEM). No significant differences were found in mean change between BC and MBC. The dashed red line denotes the competition.

Figure 7: Assessing correlations between A) $\Delta$ total body water (TBW) and $\Delta$ lean body mass (LBM); B) $\Delta$ intracellular fluid (ICF) and $\triangle \mathrm{LBM} ; \mathrm{C}) \Delta$ extracellular fluid (ECF) and $\triangle \mathrm{LBM}$ significant relationships were found between $\Delta$ TBW and $\Delta$ LBM $(p=.04)$, and $\Delta$ ECF and $\Delta$ LBM $(p=.01)$. NS was found for $\Delta$ ICF and $\triangle$ LBM.

Hydration Status

Figure 8: The time course analysis of 20-week contest preparation of hydration status assessed with urine specific gravity (USG) for A) Contest Preparation (20 weeks). The time course analysis was accompanied with assessment of mean average (mean \pm SEM) over 20-weeks. A significant mean USG difference $\left.{ }^{*} p=.01\right)$ was found between BC $(1.021 \pm .001 ; 95 \% \mathrm{Cl}: 1.018-1.024)$ and MBC $(1.025 \pm .001$; $95 \% \mathrm{Cl}: 1.023-1.027)$. (B) Competition Week time course analysis assessed with USG over $5 \mathrm{~d}$ (D1-D5). NS was found between BC and MBC. The dashed red line and circle denotes the competition. The solid blue line denotes the euhydration threshold $\left(1.020 \mathrm{~g} \cdot \mathrm{mL}^{-1}\right)$.

Resting Metabolic Rate 
Figure 9: Exploratory assessment of the time course of RMR during 20-week contest preparation with accompanying mean average (mean \pm SEM) comparison between $B C$ and MBC. A) No RMR differences were found between $B C$ and $M B C$ at any time after normalizing to $\mathrm{kcal}^{\circ} \mathrm{kgLBM}^{-1} \cdot \mathrm{d}^{-1}$. There was a difference (** $p=.005)$ found between BC (38.01 $\left.\pm 0.38 \mathrm{kcal}^{*} \mathrm{kgLBM}^{-1} \cdot \mathrm{d}^{-1} ; 95 \% \mathrm{Cl}: 37.01-39.01\right)$ and MBC $\left(35.35 \pm 0.64 \mathrm{kcal} \cdot \mathrm{kgLBM}^{-1} \cdot \mathrm{d}^{-1} ; 95 \% \mathrm{Cl}: 33.69-37.01\right)$. The red dashed line denotes the competition.

\section{Energy Intake and Exercise Training Recall}

Figure 10: The time course analysis of 20-week contest preparation for energy intake from the selfreported 4-day dietary recall with accompanying mean average (mean \pm SEM) comparison between BC and MBC. A) A difference ( $\left.{ }^{* \star \star} p=<.0001\right)$ was found in energy intake between BC (1791 $\pm 80.45 \mathrm{kcal}^{-\mathrm{d}^{-1}}$; $95 \% \mathrm{Cl}: 1612-1912)$ and MBC (1137 $\left.\pm 42.35 \mathrm{kcal} \cdot \mathrm{d}^{-1} ; 95 \% \mathrm{Cl}: 1043-1232\right)$. B) A difference ( ${ }^{\star \star \star \star} p=$ $<.0001)$ was found in energy intake between $\mathrm{BC}\left(34.18 \pm 1.85 \mathrm{kcal} \cdot \mathrm{kg}^{-1} \cdot \mathrm{d}^{-1} ; 95 \% \mathrm{Cl}: 30.04-38.31\right)$ and MBC (22.25 $\left.\pm 0.68 \mathrm{kcal} \cdot \mathrm{kg}^{-1} \cdot \mathrm{d}^{-1} ; 95 \% \mathrm{Cl}: 20.71-23.78\right)$. C) A difference ( $\left.{ }^{\star *} p=.001\right)$ was found in energy intake between $\mathrm{BC}\left(43.20 \pm 3.24 \mathrm{kcal} \cdot \mathrm{kgLBM}^{-1} \cdot \mathrm{d}^{-1} ; 95 \% \mathrm{Cl}: 25.26-31.01\right)$. The red dashed lines denote competition.

Figure 11: The time course analysis of the 20-week contest preparation macronutrient intake from the self-reported 4-day dietary recall with accompanying mean average (mean \pm SEM) comparison between

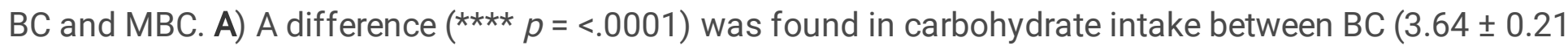
$\left.\mathrm{g} \cdot \mathrm{kg}^{-1} \cdot \mathrm{d}^{-1} ; 95 \% \mathrm{Cl}: 3.16-4.12\right)$ and $\mathrm{MBC}\left(1.354 \pm 0.15 \mathrm{~g} \cdot \mathrm{kg}^{-1} \cdot \mathrm{d}^{-1} ; 95 \% \mathrm{Cl}: 1.00-1.70\right)$. B) A difference (* $p=$ $0.0163)$ was found in protein intake between $\mathrm{BC}\left(2.96 \pm 0.07 \mathrm{~g} \cdot \mathrm{kg}^{-1} \cdot \mathrm{d}^{-1} ; 95 \% \mathrm{Cl}: 2.80-3.13\right)$ and MBC $(2.72$ $\left.\pm 0.05 \mathrm{~g} \cdot \mathrm{kg}^{-1} \cdot \mathrm{d}^{-1} ; 95 \% \mathrm{Cl}: 2.60-2.84\right)$. C) A difference ( ${ }^{\star \star} p=0.009$ ) was found lipid intake between BC $\left(0.91 \pm 0.10 \mathrm{~g} \cdot \mathrm{kg}^{-1} \cdot \mathrm{d}^{-1} ; 95 \% \mathrm{Cl}: 0.6822-1.156\right)$ and MBC (0.58 $\left.\pm 0.04 \mathrm{~g} \cdot \mathrm{kg}^{-1} \cdot \mathrm{d}^{-1} ; 95 \% \mathrm{Cl}: 0.4835-0.6910\right)$. The red dashed line denotes competition.

Figure 12: Time course analysis during 16-week pre-contest preparation prior to competition of selfreported resistance training (RT) volume load (sets $\times$ reps $\times \mathrm{kg}$ ) and aerobic training (AT) volume (min $\times \mathrm{d})$ with accompanying mean average (mean \pm SEM) comparison between BC and MBC. A) A difference was found ( $\left.{ }^{*} p=.01\right)$ in the mean total RT volume load between BC $(6669 \pm 256.1 ; 95 \% \mathrm{Cl}: 6078-7,259)$ and MBC (8879 $\pm 752.0 ; 95 \%$ Cl: 7100-10657). B) A difference ( $\left.{ }^{*}=.03\right)$ was found between mean upper body (UB) volume load between BC (4921 \pm 189.6 ; $95 \% \mathrm{Cl}$ : 4484-5358) and MBC (5477 $\pm 141.9 ; 95 \% \mathrm{Cl}$ : 5142-5813). C) A difference ( $p=.01)$ was found between mean lower body (LB) volume load between BC (8909 $\pm 476.9 ; 95 \% \mathrm{Cl}: 7809-10009)$ and MBC (12987 $\pm 1470 ; 95 \% \mathrm{Cl}: 9421-16373)$. D) A difference ${ }^{*} p=$ .03) was found in mean AT volume between BC (198.9 \pm 31.84 ; $95 \%$ Cl: $125.5-272.3)$ and MBC (105.6 \pm 23.95; 95\% Cl: 48.99-162.3).

\section{Hormone Analysis}

Figure 13: Time course analysis during 16-week pre-contest preparation of reproductive and metabolic hormones with accompanying mean differences (mean \pm SEM). A) No mean difference (ns) in Estradiol 


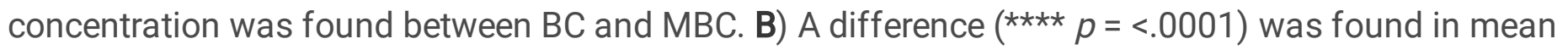
luteinizing hormone $(\mathrm{LH})$ concentration between $\mathrm{BC}\left(3.66 \pm 0.23 \mathrm{IU} \cdot \mathrm{L}^{-1} ; 95 \% \mathrm{Cl}: 3.00-4.31\right)$ and MBC (88.34 $\left.\pm 6.01 \mathrm{IU} \cdot \mathrm{L}^{-1} ; 95 \% \mathrm{Cl}: 71.65-105.0\right)$. C) A difference (* $\left.p=.04\right)$ was found in triiodothyronine $\left(\mathrm{T}_{3}\right)$ between BC (122.9 $\left.\pm 6.46 \mathrm{ng} \cdot \mathrm{dL}^{-1} ; 95 \% \mathrm{Cl}: 105.0-140.9\right)$ and MBC $\left(93.64 \pm 10.75 \mathrm{ng} \cdot \mathrm{dL}^{-1} ; 95 \% \mathrm{Cl}: 63.78-\right.$ 123.5).

Figure 14: Time course analysis during 16-week pre-contest preparation of energy balance hormones with accompanying mean differences (mean \pm SEM). A) A mean difference ( ${ }^{\star \star \star} p=.008$ ) in ghrelin concentration was found between BC (91.63 $\left.\pm 8.14 \mathrm{pg} \cdot \mathrm{mL}^{-1} ; 95 \% \mathrm{Cl}: 69.01-114.2\right)$ and MBC (40.05 \pm 5.71 $\left.\mathrm{pg} \cdot \mathrm{mL}^{-1} ; 95 \% \mathrm{Cl}: 24.18-55.92\right)$. B) A difference (**** $\left.p=.005\right)$ was found in mean leptin concentration between BC (3.61 $\pm 0.17 \mathrm{ng} \cdot \mathrm{mL}^{-1} ; 95 \% \mathrm{Cl}$ : 3.13-4.09) and MBC (25.55 $\pm 0.98 \mathrm{ng} \cdot \mathrm{mL}^{-1} ; 95 \% \mathrm{Cl}$ : 22.81-

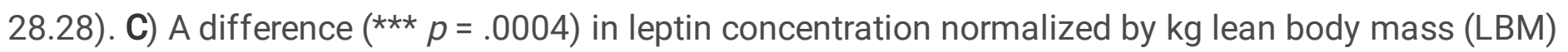
between $\mathrm{BC}\left(0.37 \pm 0.03 \mathrm{ng} \cdot \mathrm{mL}^{-1} \cdot \mathrm{kgLBM}^{-1} ; 95 \% \mathrm{Cl}: 0.28-0.47\right)$ and $\mathrm{MBC}\left(2.89 \pm 0.24 \mathrm{ng} \cdot \mathrm{mL}^{-1} \cdot \mathrm{kgLBM}^{-1}\right.$; $95 \%$ Cl: 2.22-3.56).

Psychometrics

Figure 14: The time course analysis of the impact of a 20-week contest preparation on body image and perceived stress with accompanying mean average (mean \pm SEM) comparison between BC and MBC. A) A mean difference ( ${ }^{\star} p=0.03$ ) was found in the perceived stress scale (PSS) between BC (8.09 \pm 1.09 ; $95 \% \mathrm{Cl}: 5.66-10.5)$ and MBC (11.64 $\pm 1.17 ; 95 \% \mathrm{Cl}$ : 9.03-14.24). B) A mean difference ( $* \star \star p=0.0003)$ was found in the body image satisfaction scale (BISS) between BC (44.00 $\pm 0.75 ; 95 \%$ Cl: 42.33-45.67) and MBC (38.00 $\pm 1.16 ; 95 \% \mathrm{Cl}: 35.40-40.60)$. The red dashed line denotes competition.

Figure 15: The psychometric analysis of the baseline and pre-competition on the $\mathrm{A}$ ) body appreciation scale (BAS), B) EAT-26, and C) social physique anxiety scale (SPAS) comparison between BC and MBC.

\section{Discussion}

Over the 16-week pre-contest preparation, as expected, both competitors lost roughly $4 \mathrm{~kg}$ of bodyweight, which was predominantly explained by a loss of $\sim 3 \mathrm{~kg}$ of fat mass (FM) and $\sim 3 \%$ body fat (BF\%). The bikini competitor's $(\mathrm{BC})$ baseline value or starting point for both $\mathrm{BF}$ and $\mathrm{BF} \%$ were both higher than the master's bikini competitor (MPC) comparatively. However, the BC's BF\% change was much greater ( 19\%). This is concurrent with the BC's greater reduction in ultrasound (US) assessed total subcutaneous adipose tissue (SAT) values with a $-34 \%$ reduction from baseline. Hulmi, et al. (2017) observed a $\sim 7 \mathrm{~kg}$ fat mass loss from a higher fat mass baseline value $(\sim 14 \mathrm{~kg})$ in their categorical heterogenous female-physique population, which was more than double than seen in this bikini-physique categorical population. However, due to the heterogenic population assessed, it is difficult to determine the results for the bikini-physique competitors alone. From our perspective, this may be explained in that this bikini-physique competitor's maintain higher BF\% during their normal, non-contest preparation time 
periods than other notable female-physique competitors and the differing judging criteria, which may determine the female-physique competitor's goal of required fat mass.

Prior to competing, and at their lowest fat mass assessment, both $\mathrm{BC}$ and $\mathrm{MBC}$ maintained their $\mathrm{BF} \%$ greater than $12 \%$ ( 14 \%), which is the recommended threshold for female athletes in weight sensitive competition and sports to reduce risks of health defects ${ }^{48}$. Low BF\% mixed with high energy expenditure and very low kilocalorie intake would lead to a LEA status. This has shown to negatively affect menstrual function, and bone mineral density, which may have clinical manifestations including eating disorders, functional hypothalamic amenorrhea, and osteoporosis known as "The Female Athlete Triad" ${ }^{49}$. In assessing both competitor's lean body mass (LBM) and interrelated skeletal muscle mass (SKMM) measures, both were well preserved throughout their contest preparation ( $\angle \mathrm{D}-0.5$ and $-2.1 \%$ respectively). It is well known that resistance training (RT) is a potent stimulator of muscle protein synthesis and muscle hypertrophy and concurrent with an energy restricted state reduces LBM losses ${ }^{50,51}$. Additionally, preservation of LBM in athletes is further increased with the combination of RT and higher protein

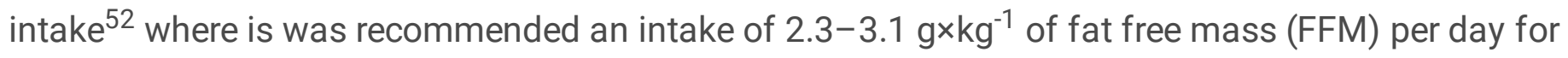
energy restricted, resistance training athletes ${ }^{53}$. Both the $B C$ and $M B C$ competitors reported a very high

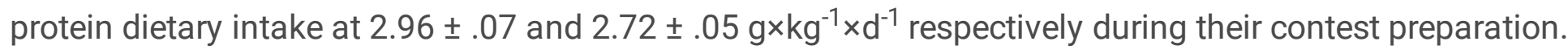

Both the BC and MBC showed fairly stable MF-BIA measured TBW, ICF, and ECF measures during the contest preparation. The BC showed a higher value of ICF fluid, which is most likely explained by a higher $\mathrm{kg} \mathrm{LBM}$ and it is well identified that $\sim 60 \%$ of human TBW is stored intracellular and represents $70-75 \%$ of LBM. We did not observe any notable change in TBW, ICF, and ECF measures $5 \mathrm{~d}$ post-competition in either the $\mathrm{BC}$ or the MBC. We did not acquire any dietary recall data post-competition. If the $\mathrm{BC}$ and $\mathrm{MBC}$ indulged in high kilocalorie, post-competition hyperphagia ${ }^{26}$, it did not impact immediate TBW measures.

Our exploratory US measures, while not validated, did see some changes from baseline worth evaluating and interpreting due to their relative importance to the population assessed. In analyzing the deltoid (Delt $\left.{ }_{C S A}\right)$ muscle group in both competitors we saw an average increase in CSA ( $\left.D+4.63 \%\right)$ and reduction in gluteus maximus muscle thickness $\left(\mathrm{GM}_{\mathrm{MT}}\right)$ muscle thickness ( $\left.\mathrm{D}-19.5 \%\right)$. This dichotomy in muscular response is rather perplexing yet may be able to be explained by both exercise training and energy intake. Interestingly, both the $\mathrm{BC}$ and $\mathrm{MBC}$ competitors self-reported a higher amount exercise training stimulus of the lower body. Using the Stairmill/Stairmaster as their primary mode of aerobic training during their contest preparation and when averaged, both competitors reported a higher lower body (LB) than upper body (UB) resistance training volume (UB: 5,199; LB: 10,903 set $\cdot r e p s \cdot k g)$. The gluteus maximus (GMax) is the largest muscle of the hip accounting for $16 \%$ of the total CSA in the region. This muscle group is often used to accelerate the body upward and forward from a position of hip flexion ranging from $45^{\circ}$ to $60^{\circ}$ (e.g., pushing off into a sprint, arising from a deep squat, or climbing a very steep hill). Recently, it has been shown that the step-up exercise had the highest GMax myoelectrical activity (169.22 $\pm 101.47 \% \mathrm{MVIC}$ ) in comparison to other known hip exercises ${ }^{54}$. However, the BC and MBC competitors

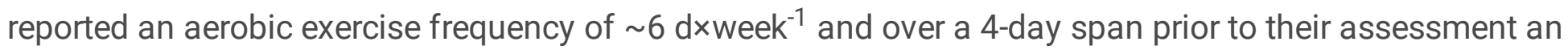


average of $198.9 \pm 31.8(95 \% \mathrm{Cl}: 125.5-272.3)$ and $105.6 \pm 23.9(95 \% \mathrm{Cl}: 48.99-162.3)$ min respectively where $\mathrm{BC}$ had a higher volume of AT $(p=.003)$. Although speculative, $\mathrm{GM}_{\mathrm{MT}}$ reduction or atrophy may likely be more related to local glycogen and fluid loss than muscle protein loss due to restricted

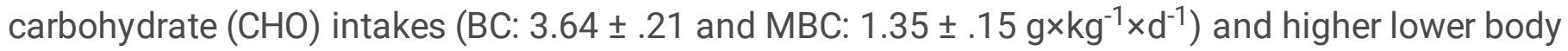
exercise training volume. Both the $\mathrm{BC}$ and $\mathrm{MBC}$ competitors self-reported a higher mean lower body (LB) compared to upper body (UB) RT volume loads (BC-LB: $8909 \pm 476.9$ vs BC-UB: $4921 \pm 189.6$

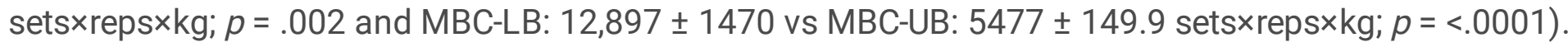
To support fluid loss as a potential factor and a plausible explanation, in Figure7 we compared monthly $D$ change with dual X-ray absorptiometry (iDXA) LBM (g), FM (kg) changes, and bioimpedance analysis (MF-BIA) configured total body water (TBW), extracellular fluid (ECF), and intracellular fluid (ICF) compartment changes $(\mathrm{mL})$ over the 16-week pre-contest preparation period. Relationships between both LBM and TBW $\left(r=.64 ; r^{2}=.41 ; p=.04\right)$, and ECF $\left(r=.72 ; r^{2}=.52 ; p=.01\right)$ were found. No relationships were found between LBM and ICF changes, and no relationship was found between FM and TBW changes. Moreover, although no difference was found between competitors, both $\mathrm{BC}$ and $\mathrm{MBC}$ showed an average ECF fluid loss over the 16-week period. It has been shown that short term hydration and muscle glycogen status may influence DXA-LBM measures ${ }^{55-57}$. Due to the inability of DXA to differentiate between ICF and ECF compartments, it is feasible that the $\mathrm{GM}_{\mathrm{MT}}$ reduction may be potentially explained by local glycogen and fluid loss from higher LB exercise training volumes and lower $\mathrm{CHO}$ intake.

The exploration of utilizing US to assess SAT measures and changes compared to iDXA, we found that time course changes in the sum total of 7-site SAT measures correlated well with iDXA $\left(r=.81 ; r^{2}=.66 ; p\right.$ $=.001)$ FM measures. Additionally, when estimating BF\% using Jackson and Pollock ${ }^{31} 7$-site equation, we found US acquired SAT measures correlated well with iDXA configured BF\% $\left(r=.78 ; r^{2}=.60 ; p=.002\right)$. The US method has been utilized for SAT measures by Trexler, et al. (2017) that assessed physique athletes utilizing an automated program that estimated $\mathrm{BF} \%{ }^{5}$. However, we found no relationship between iDXA derived $\mathrm{kg}$ FM and US-SAT mm D changes. Utilizing B-mode US as a modality to investigate SAT changes may have some practical use for individuals that do not have access to other expensive compositional measures to assess $\mathrm{BF} \%$.

No differences were observed between the $\mathrm{BC}$ and $\mathrm{MBC}$ total body water changes throughout the 20-week observational period. The MBC's TBW saw a very slight reduction $(-1.3 \% \mathrm{D})$ compared to baseline values when comparing baseline to week 16 . Additionally, we sought to assess both the BC's and MBC's TBW changes $5 \mathrm{~d}$ post-competition upon their return to the laboratory. Due to long periods of low energy status coupled with repetitive dietary choices and increased hunger may lead to immediate post-competition hyperphagia or binge eating ${ }^{58}$ with an acute response similar to what may be seen with carbohydrate loading schemes used by endurance athletes after a glycogen depletion phase. Typically, glycogen is stored in a 1:3-5 ratio with water ${ }^{59}$, which may lead to changes seen in weight gain and fluid perturbations post-competition. Both the $\mathrm{BC}$ and $\mathrm{MBC}$ saw no significant increases of either weight or TBW alterations 5-d post competition. 
In our observation of BMD, we did not expect to see any notable changes ( $£ 1 \% \mathrm{D}$ change) in bone mineral density (BMD) or bone mineral content (BMC) over the 16-week contest preparation time period due the incremental effect of exercise on BMD to be very slow $(6-12 \mathrm{mo})^{60}$. It should be noted, while no significant BMD changes were observed, both $B C$ and $\mathrm{MBC}$ maintained $>100 \%$ of their age-related Z-score with lower observed E2 range levels. Further investigations should isolate the impact of exercise traininginduced mechanotransductive stress compared to LEA-induced inhibition on reproductive hormone concentration and their integrated longitudinal effect on BMD in females.

During the 16-week contest preparation, both the $\mathrm{BC}$ and $\mathrm{MBC}$ maintained a mean categorical "significant

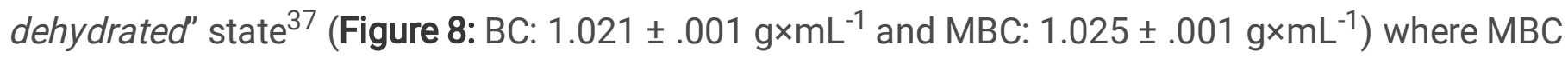
averaged to be slightly more dehydrated than $\mathrm{BC}(p=.01)$. However, from a practical aspect, it is unknown how meaningful this slight difference may be. It should be noted that each competitor was asked to visit the laboratory after a $\sim 8 \mathrm{~h}$ fast and prior to ingesting any food or drink. This USG assessment may not be indicative of their behaviors throughout the rest of the day and between visits. It is interesting after the week 12 , the $B C$ 's hydration status moved below the $1.020{\mathrm{~g} \times \mathrm{mL}^{-1} \text { euhydration }}^{38}$, which is a status of euhydration ${ }^{61}$. To our knowledge, we have not observed any previous literature examining hydration status of competitors during their competition week. During this time period there may be manipulation of fluid consumption by restricting water intake ${ }^{62}$ and/or pharmacologically induced fluid excretion through the use of diuretics ${ }^{63}$ with the purpose of reducing fluid content that may influence their ability to present muscular detail to the judging panel. Notably, due the judging criteria for bikini-physique competitions, these water manipulating procedures may not be as aggressively used due to less focus on muscularity and conditioning. During competition week (D1-D5), comparatively, both the

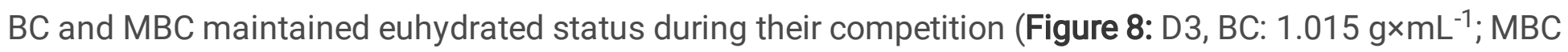
$1.018{\mathrm{~g} \times \mathrm{mL}^{-1}}^{-1}$ respectively). After the competition, the $\mathrm{BC}$ maintained a euhydrated status while the MBC's values elevated closer to the average. This contrast in hydration status may be explained that the $\mathrm{BC}$ competitor chose not to compete again after this competition in comparison to the MBC that chose to compete again a few weeks later.

During the competition preparation was saw a slight reduction in resting metabolic rate (RMR) at the 4week time point for the BC. This may be explained by self-reported reduction in kilocalorie intake and changes in body composition. Overall, during the contest preparation, RMR was fairly stable even with their body weight reduction where both $B C$ and $M B C$ showed mean positive $D$ RMR change value. Due to that the majority of both BC's and MBC's weight loss being attributed to FM loss, we sought to analyze any relationship between change in D FM and D RMR. We found no correlation between contest preparation D FM change and D RMR change. However, it has been shown and readily accepted that the loss of both FM and LBM will impact RMR ${ }^{64}$. Additionally, in observing the active thyroid hormone triiodothyronine $\left(T_{3}\right)$ with its known relationship with metabolism, we observed no significant correlation with $\mathrm{D} \mathrm{T}_{3}$ and $\mathrm{D}$ RMR change. In our results comparing baseline to week 16 , we observed that both $\mathrm{BC}$ and $\mathrm{MBC}$ had a reduction in $\mathrm{T}_{3}$ hormone concentration of $\sim 14 \%$ and $35 \%$ respectively. It is well established that thyroid hormone status regulates energy expenditure and therefore a factor in 
bodyweight changes ${ }^{65}$. Additionally, it has been shown that basal metabolic rate (BMR), which is $~ 10 \%$ lower than RMR is highly correlated with lean body mass. Being that LBM was mostly stable in both BC and $\mathrm{MBC}$ during contest preparation, this may suggest these competitor's RMR was maintained by the factor of LBM more so than the observed $T_{3}$ reduction. However, this is merely speculation being that none of these variables were assessed and isolated directly. Lastly, when comparing the BC and MBC competitors (Figure 9), we found RMR differences between time points. However, when RMR values were normalized per kg LBM compared, a small difference $\left(\sim 2.6 \mathrm{kcal}^{\circ} \mathrm{kgLBM}^{-1} \cdot \mathrm{d}^{-1}\right)$ was found where BC was had a slightly higher RMR. This outcome was not entirely surprising in that there have been reports that a decline in RMR is associated with age. However, physically active older adults that maintain similar exercise training volume and energy intake maintain a similar RMR ${ }^{66}$. Moreover, it is known that LBM is highly correlated with RMR; however, visceral organ tissue is more metabolically active than is skeletal muscle tissue during resting conditions, which may explain some the variances in $\mathrm{RMR}^{67}$. Therefore, in future studies assessing and comparing RMR in physique athletes, normalizing to LBM may reduce inherent variability. Lastly, it should be noted that the BC's RMR increased post-competition at 20-week, after self-reporting following a "reverse dieting" protocol, a slight increase in FM $(\sim 1.1 \mathrm{~kg})$ and reducing exercise training frequency and volume. The MBC continued a contest preparation regimen for another competition.

Our goal in observing endocrine responses in these bikini-physique competitors, was to determine if age may have played a role in the responses to a restricted energy intake and increased energy expenditure. In our observation, estradiol (E2) and luteinizing hormone (LH) remained fairly stable for BC during contest preparation, with little variation in concentration from baseline to week 12. Both E2 and LH baseline values were near the lower end of both normal ranges. After the $12^{\text {th }}$ week of the BC's contest preparation, we observed a $-35 \%$ reduction of E2 at week 16, prior to competition. This concentration level fell below $\left(\sim 6 \mathrm{pg} \times \mathrm{mL}^{-1}\right)$ what is considered the normal range relative to both follicular, mid-cycle, and luteal phases (10-300 pg $\left.\times \mathrm{mL}^{-1}\right)$. However, the BC's mean E2 concentration over the 16-week contest preparation was

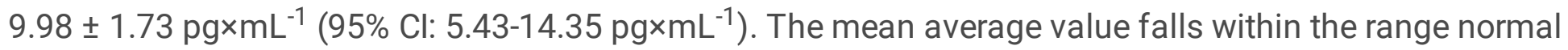
found in postmenopausal females $\left(<10 \mathrm{pg} \times \mathrm{mL}^{-1}\right)$. Observing the BC's LH time course, while relatively

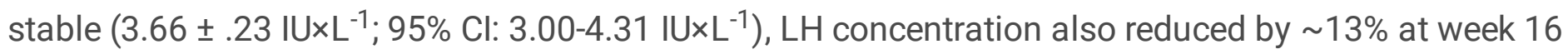
prior to the competition.

Comparatively, the MBC's assessed E2 concentrations were more variable during the 16-week contest preparation. With the respect the MBC's age status of $44 \mathrm{y}$ during this case series, which is close to the age of $45 \mathrm{y}$ that has been shown in cross-sectional studies when endocrine changes and the onset of the perimenopause begin ${ }^{68}$. The MBC's E2 concentrations reduced $24 \%$ from baseline values to week 16 , prior to the competition. The mean average concentration during the 16-week pre-contest preparation was

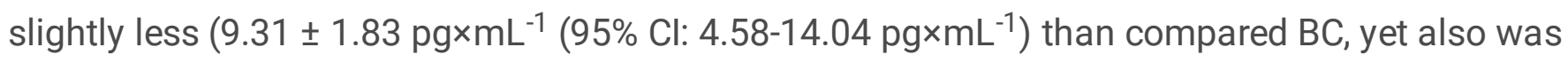
observed below the normal concentration value found in postmenopausal women $\left(<10 \mathrm{pg} \times \mathrm{mL}^{-1}\right)$. 
The MBC's LH time course concentration values seemed fairly stable until week 12 where there was a decline. There was an observed 14\% reduction in LH when comparing baseline $\left(95.9 \mathrm{IU} \times \mathrm{L}^{-1}\right)$ and week 16 values (72.02 IU $\left.\times \mathrm{L}^{-1}\right)$. Interestingly, the MBC's mean LH values over the 16-week pre-contest preparation were much higher than the $\mathrm{BC}(88.3 \pm 13.4 \mathrm{vs} 3.66 \pm .23) \mathrm{IU} \times \mathrm{L}^{-1}$ respectively). This may be expected when taking into consideration that in early perimenopause, minor elevations in LH become evident ${ }^{69}$.

In a seminal article by Loucks, et al. (1998) the "energy availability hypothesis" explained that LEA from low energy intake and high energy expenditure may inhibit gonadotropin-releasing hormone $(\mathrm{GnRH})^{70}$, which is derived from $\mathrm{GnRH}$ nerves located in the hypothalamic-pituitary-gonadal axis (HPTA) that is a pulse generator that controls the pulsatile secretion of the gonadotropic hormone LH, which is critical for reproduction ${ }^{71}$. Based on the $\mathrm{BC}^{\prime}$ s dietary recall, the average energy intake over the 16-week pre-contest preparation was $43.2 \pm 3.2 \mathrm{~g} \mathrm{kgLBM}^{-1} \times \mathrm{d}^{-1}$. This value meets the recommended energy intake range requirement shown to maintain $\mathrm{LH}$ pulsatility ${ }^{16}$. However, due to the inability to accurately capture energy expenditure during this case series and the subtracting that value from energy intake, it is feasible to suggest that the actual energy availability may be lower than the estimated energy intake. In comparison, the MBC's mean average, self-recalled dietary intake was $28.1 \pm 1.1 \mathrm{~g} \times \mathrm{kgLBM}^{-1} \times \mathrm{d}^{-1}$, which is below the recommended range to maintain LH pulsatility. Additionally, the self-reported value does not take into consideration energy expenditure from exercise training. The energy availability may actually be lower for the MBC. In contrast to the Louck's suggests that LEA inhibits LH pulsatility, the higher LH concentrations observed during the MBC's contest preparation may suggest that perimenopausal-induced LH increases may supersede LEA status inhibition of LH pulsatility. Furthermore, it should be noted that the majority of the work assessing LEA on female reproductive systems, has been investigated in younger, female athletes $(<29 \mathrm{y})$. We feel this is a fairly novel finding that requires much more investigation to determine how LEA may impact the reproductive system and metabolism in female athletes near perimenopause status.

Additional to assessing endocrine hormones related to reproduction and metabolism, we also investigated the impact of contest preparation on leptin and ghrelin. Leptin has been reported to have influence on various biological mechanisms such as initiating reproductive hormones, menstruation, regulatory centers in the brain to inhibit food intake and to regulate bodyweight and energy homeostasis $^{72}$. Leptin is primarily synthesized and secreted from adipocytes in white adipose tissue and is normally found in higher blood concentrations in persons with higher BMI and BF\%. Additionally, factors such as hyperglycemia and hyperinsulinemia also facilitate leptin secretion. However, in contrast, factors that are related to inhibiting leptin release are increasing age ( $\left.{ }^{3} 40 \mathrm{y}\right)^{72}$. In our investigation, we found that the BC's leptin concentrations reduced $4 \%$ from baseline values at the 16 -week time point. The BC's mean average leptin concentration during pre-contest preparation fell slightly below the normal

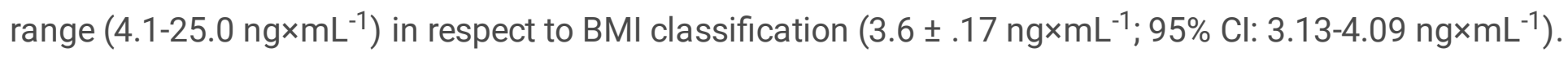
The BC's baseline leptin level was near the lower range normally found; however, this may be explained by a lower BF\% compared to non-athlete females. The MBC's mean average leptin levels were also within the 
normal range relative to $\mathrm{BMI}\left(22.8 \pm .98 \mathrm{ng} \times \mathrm{mL}^{-1} ; 95 \% \mathrm{Cl}: 22.81-28.28 \mathrm{ng} \times \mathrm{mL}^{-1}\right)$ yet were on the on the higher end of the normal scale compared to the BC. The MBC's leptin levels time course were relatively stable during pre-contest preparation. Comparing baseline to the 16-week time period, there was a small $\sim 1 \%$ increase found. This outcome was very intriguing in that the similar to the $\mathrm{BC}$, the MBC also lost $\mathrm{BF} \%$ from baseline to 16-weeks, yet this loss of fat mass did not seem to dictate leptin concentrations. This outcome is in contrast to the Longstrom, et al. (2020) who observed leptin concentrations that were responsive to fat mass changes. However, it should be noted that the female-physique competitors observed in this study were $\sim 29 \mathrm{y}^{8}$. It may be plausible that there is a link between the elevated leptin and LH concentrations we observed in the MBC. It has been investigated in previous research that increased leptin appears to drive the reproductive system through both the HPTA and GnRH-stimulated LH secretion. Therefore, the increase seen in both leptin and LH in the MBC may be interrelated. Leptin directly stimulates ovarian steroidogenesis ${ }^{73}$, yet, the E2 concentrations seemed to be less affected compared to LH found in the MBC. In our observation, it appears there may be some contrasting hormonal interrelationships between fat mass loss, leptin, LH, and E2 in our observations of the MBC compared to previously investigations that are typically seen in younger, female athletes.

Concurrent with assessing leptin responses in both $\mathrm{BC}$ and $\mathrm{MBC}$, we sought to observe any differences on the hormone ghrelin, which is an orexigenic gut peptide. The fasted elevation of ghrelin levels and its decline after food ingestion led to its relevance as a 'hunger' hormone responsible for meal initiation, which is involved in short-term regulation of food intake and long-term regulation of bodyweight through decreasing fat utilization ${ }^{74}$. Ghrelin has an impact on numerous physiological functions, although our focus was to observe any interrelationships with food intake and energy metabolism. In our observation of the BC's ghrelin response was fairly stable throughout the 16-week pre-contest preparation. There was a notable $-36 \%$ drop in the BC's ghrelin measure at week 4 . This may be explained by the $\sim 59 \%$ increase $\left(\mathrm{kcal} \times \mathrm{kgLBM}^{-1} \times \mathrm{d}^{-1}\right)$ that was self-reported from baseline to week 4 due the ghrelin secretion being regulated by nutritional status. Interestingly, the BC's mean ghrelin hormone concentration levels remained higher than the $\mathrm{MBC}$ throughout the 16-week pre-contest preparation (BC: $91.6 \pm 8.1 \mathrm{vs} \mathrm{MBC:}$ $40.0 \pm 5.7 \mathrm{pg} \times \mathrm{mL}^{-1} ; p=.0008$ ) concurrent with a higher mean kcal $\times \mathrm{kgLBM}^{-1} \times \mathrm{d}^{-1}$ than the MBC. The MBC's ghrelin level increased from 70\% from week 4 to week 8 . However, there were no changes in selfreported dietary intake $\left(\mathrm{kcal} \times \mathrm{kgLBM}^{-1} \times \mathrm{d}^{-1}\right)$. Age is a factor that influences ghrelin secretion, which may assist in explaining differences seen. However, the variations at certain time points may have other confounding factors influencing ghrelin concentration outside the parameters of our study. Our ghrelin findings and interpretations should be considered with much caution. It should be noted that the concentrations found in these bikini-physique competitors that were assessed using ELISA analysis were much lower than previous work assessing total ghrelin (both active acyl-ghrelin and inactive des-acylghrelin) hormone with similar ELISA methodology (baseline $\left.\sim 500 \mathrm{pg} \times \mathrm{mL}^{-1}\right)^{75}$ and differing

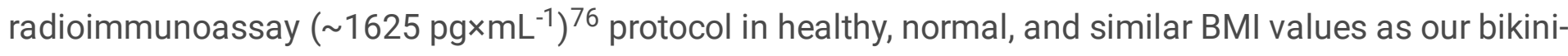
physique competitors. Additionally, another factor that may explain such low values we observed is the half-life of acyl-ghrelin in human plasma without a stabilizer or deacylation inhibitor. Per our 
methodology, we used $\mathrm{K}_{2}$ EDTA vacutainer tubes for blood collection and stored plasma samples in -80 ${ }^{\circ} \mathrm{C}$ environment. However, previous investigations showed that fasting levels of plasma derived-acylghrelin collected in $\mathrm{K}_{2}$ EDTA vacutainers decreased approximately five-fold from prior storage

measurements ${ }^{77}$. Future research that would like to investigate the gut-derived 'hunger' hormone ghrelin in blood may add this protocol to standard manufacturer ELISA methodology. To maintain sample integrity, the vacutainers may be treated with 4-(2-Aminoethyl) benzenesulfonyl fluoride hydrochloride (AEBSF) to reduce the degradation of ghrelin ${ }^{77}$ to improve accuracy of results.

In our psychometric findings, we observed that the MBC showed to have a higher mean average score of perceived stress (PSS) and a lower mean average score in the body image satisfactory (BISS) when compared to the BC. With notable environmental (e.g., lifestyle, competition experience) and inherent psychological factors that could influence these measures, it is unknown if age has an may have impacted these responses. The BAS- 2 assessment pre, and post-contest preparation was stable for BC. In comparison, the MBC's response declined from baseline to post-contest preparation. This difference found may be partially explained in that the MBC continued contest preparation for another competition while the BC elected to progress into a "off-season" status. The eating attitude analysis (EAT-26) showed both $B C$ and $M B C$ increase post-competition when compared to baseline. This difference may be partially explained and influenced by the post-competition hyperphagia. Lastly, the measures of social anxiety both increased from baseline to post-contest in the BC and MBC. The anxiety related to contest performance may play a role in this assessment.

\section{Conclusion}

Our case series investigation of the $32 \mathrm{y}$ and the master's $44 \mathrm{y}$ bikini-physique competitors during a 16week pre-contest preparation observed that their adaptations were fairly similar in that no differences found in musculoskeletal or body water changes during pre-contest preparation. The hormonal differences seen may be explained due to a difference in age being the middle-aged bikini-physique competitor may be near perimenopausal status. Hydration status during pre-contest preparation was considered to be mainly in a dehydrated state for both competitors. Both seemed to become more hydrated as the competition date became closer and maintained a positive hydration status on the day of the competition. There were notable volume differences in training protocols; however, this inter-variability could be expected between competitors. Similarly, dietary regimens and energy intake did not did not fall within the recommended ranges, which seems to be a normal response in investigations observing female-physique competitors ${ }^{3,7,11}$. The exploratory protocols used to assess skeletal muscle changes in the bikini-physique competitors have not been validated for accuracy. However, the regional muscle groups assessed may hold a more relative reference value for bikini-physique competitors than the validated measures of vastus lateralis ${ }^{32,33,78}$. With the understanding the limitations of this case series investigation, the hormonal leptin and LH outcomes that were observed in the master's bikini-physique competitor that elevated regardless of reduced body fat and low energy status should be further investigated in similar female demographic populations. This outcome was in contrast to what has been 
previously seen in younger female athletes during $\operatorname{LEA}^{16,70,79}$ and other case studies observing leptin changes female-physique competitors ${ }^{8}$. With the increased popularity of bikini-physique competition and the similar compositional adaptations seen in the master's bikini-physique competitor compared to the younger bikini-physique competitor, more studies should recruit and observe competitors in the master's division as this may assist other females in the demographical area to engage in exercise and nutritional training protocols that may minimize the known physiological and metabolic changes associated with menopause transition.

\section{Abbreviations}

IFBB - International Federation of Bodybuilding

NPC - National Physique Committee

BMR - Basal metabolic rate

$\mathrm{BMI}$ - Body mass index

BMD - Bone mineral density

$\mathrm{CHO}$ - Carbohydrate

DXA/iDXA - Dual X-ray absorptiometry

LBM - Lean body mass

US - Ultrasound

MT - Muscle thickness

CSA - Cross-sectional area

$\mathrm{T}_{3}$ - Triiodothyronine

RMR - Resting metabolic rate

LEA - Low energy availability

EA - Energy availability

El - Energy intake

EEE - Exercise energy expenditure

MF-BIA - Multifrequency bioelectrical impedance analysis 
$\mathrm{T}_{4}$ - Thyroxine

BC - Bikini competitor

MBC - Master's bikini competitor

RT - Resistance training

UB - Upper body resistance training

LB - Lower body resistance training

AT - Aerobic training

FM - Fat mass

SAT - Subcutaneous adipose tissue

Delt $_{\text {CSA }}$ - Deltoid cross-sectional area

GMax - Gluteus maximus

$\mathrm{GM}_{\mathrm{MT}}$ - Gluteus maximus muscle thickness

TBW - Total body water

ICF - Intracellular fluid

ECF - Extracellular fluid

USG - Urine specific gravity

PSS - Perceived Stress Scale

BISS - Body Image States Scale

BAS-2 - Body Appreciation Scale

SPAS - Social Physique Anxiety Scale

EAT-26 - Eating Attitudes Test 26

SKMM - Skeletal muscle mass

$\mathrm{D}_{2} \mathrm{O}$ - Isotopic deuterium dilution

ASLT - Appendicular soft lean tissue 
SEE - Standard error of estimate

$\mathrm{MHz}$ - Mega hertz

ACSM - American College of Sports Medicine

$\mathrm{NIH}$ - National Institute of Health

ICC - Intraclass correlation coefficient

$\mathrm{CV} \%$ - Coefficient of variation (\%)

MRI - Magnetic resonance imaging

PSIS - Posterior superior iliac spine

IT - Ischial tuberosity

$\mathrm{DDH}_{2} \mathrm{O}$ - Doubly distilled de-ionized water

$\mathrm{RMR}_{\text {Meas }}-$ Measured RMR

$\mathrm{RMR}_{\text {Calc }}$ - Estimated RMR

EDTA - Ethylenediaminetetraacetic acid

WHO - World Health Organization

ELISA - Enzyme-linked immunosorbent assay

LH - Luteinizing hormone

E2 - Estradiol

GnRH - Gonadotropin-releasing hormone

HPTA - Hypothalamic-pituitary-gonadal axis

AEBSF - 4-(2-Aminoethyl) benzenesulfonyl fluoride hydrochloride

\section{Declarations}

\section{Ethics Approval and Consent to Participate}

Written informed consent was obtained from both participants and any accompanying images were approved by participants. This study was approved by the Texas A\&M University-Corpus Christi Institutional Review Board (\#TAMU-CC-IRB-2020-02-027) and the Institutional Biosafety Committee. 


\section{Consent for Publication}

Written informed consent was obtained from both participants for publication of this Case Series report and available for review by the Editor-in-Chief of this journal upon request.

\section{Availability of Data and Materials}

The datasets during and/or analyzed during the current study available from the corresponding author on reasonable request.

\section{Competing interests}

The authors declare that they have no competing interests.

\section{Funding}

Funding for the ELISA biochemistry kits was acquired from Texas A\&M University-Corpus Christi, College of Education Research Enhancement Grant.

\section{Authors' Contributions}

Both DN and HW contributed with the study design and data collection. DN and HW both assisted in data analysis and interpretation. Both DN and HW contributed to construction of the manuscript.

\section{Acknowledgements}

We would like to thank the bikini-physique competitors that committed to attending our laboratory biweekly for 20-weeks to be observed and assessed. Committing to longitudinal studies require much is indeed difficult and we are thankful for their assistance. The value of committed participants is incomparable.

\section{References}

1. Sternfeld B and Dugan S: Physical activity and health during the menopausal transition. Obstet Gynecol Clin North Am 2011, 38:537-66.

2. Grindler NM and Santoro NF: Menopause and exercise. Menopause 2015, 22:1351-1358.

3. Tinsley GM, Trexler ET, Smith-Ryan AE, et al.: Changes in body composition and neuromuscular performance through preparation, 2 competitions, and a recovery period in an experienced female physique athlete. The Journal of Strength \& Conditioning Research 2019, 33:1823-1839.

4. Whitehead J, Slater $\mathrm{G}$, Wright $\mathrm{H}$, et al.: Disordered eating behaviours in female physique athletes. European journal of sport science 2020, 20:1206-1214.

5. Trexler ET, Hirsch KR, Campbell Bl, et al.: Physiological changes following competition in male and female physique athletes: A pilot study. International journal of sport nutrition and exercise 
metabolism 2017, 27:458-466.

6. Gray J: Associations between eating disorder risk, emotional intelligence, and body image experience in bikini competitors. Keiser University; 2019.

7. Halliday TM, Loenneke JP, and Davy BM: Dietary intake, body composition, and menstrual cycle changes during competition preparation and recovery in a drug-free figure competitor: A case study. Nutrients 2016, 8:740.

8. Longstrom JM, Colenso-Semple LM, Waddell BJ, et al.: Physiological, psychological and performance-related changes following physique competition: A case-series. Journal of Functional Morphology and Kinesiology 2020, 5:27.

9. Rohrig BJ, Pettitt RW, Pettitt CD, et al.: Psychophysiological tracking of a female physique competitor through competition preparation. Int J Exerc Sci 2017, 10:301-311.

10. Petrizzo J, DiMenna FJ, Martins K, et al.: Case study: The effect of 32 weeks of figure-contest preparation on a self-proclaimed drug-free female's lean body and bone mass. Int J Sport Nutr Exerc Metab 2017, 27:543-549.

11. Mathisen TF, Heia J, Raustøl M, et al.: Physical health and symptoms of relative energy deficiency in female fitness athletes. Scandinavian Journal of Medicine \& Science in Sports 2020, 30:135-147.

12. Hulmi JJ, Isola V, Suonpää M, et al.: The effects of intensive weight reduction on body composition and serum hormones in female fitness competitors. Frontiers in Physiology 2017, 7.

13. Thomas DT, Erdman KA, and Burke LM: Position of the academy of nutrition and dietetics, dietitians of canada, and the american college of sports medicine: Nutrition and athletic performance. Journal of the Academy of Nutrition and Dietetics 2016, 116:501-528.

14. Cunningham JJ: A reanalysis of the factors influencing basal metabolic rate in normal adults. The American journal of clinical nutrition 1980, 33:2372-2374.

15. Thomas DT, Erdman KA, and Burke LM: Nutrition and athletic performance. Medicine and science in sports and exercise 2016, 48:543-568.

16. Loucks AB: Energy availability, not body fatness, regulates reproductive function in women. Exercise and sport sciences reviews 2003, 31:144-148.

17. Hulmi JJ, Isola V, Suonpää M, et al.: The effects of intensive weight reduction on body composition and serum hormones in female fitness competitors. Frontiers in physiology 2017, 7:689.

18. Cohen S, Kamarck T, and Mermelstein R: A global measure of perceived stress. Journal of health and social behavior 1983:385-396.

19. Cash TF, Fleming EC, Alindogan J, et al.: Beyond body image as a trait: The development and validation of the body image states scale. Eating Disorders 2002, 10:103-113.

20. Tylka TL and Wood-Barcalow NL: The body appreciation scale-2: Item refinement and psychometric evaluation. Body image 2015, 12:53-67.

21. Hart EA, Leary MR, and Rejeski WJ: Tie measurement of social physique anxiety. Journal of Sport and exercise Psychology 1989, 11:94-104. 
22. Garner D, Olmsted M, Bohr Y, et al.: The eating attitudes test: Psychometric features. Psychological medicine 1982, 12:871-878.

23. Hangartner TN, Warner S, Braillon P, et al.: The official positions of the international society for clinical densitometry: Acquisition of dual-energy x-ray absorptiometry body composition and considerations regarding analysis and repeatability of measures. Journal of Clinical Densitometry 2013, 16:520-536.

24. Kim J, Wang Z, Heymsfield SB, et al.: Total-body skeletal muscle mass: Estimation by a new dualenergy x-ray absorptiometry method. The American journal of clinical nutrition 2002, 76:378-83.

25. Anderson LJ, Erceg DN, and Schroeder ET: Utility of multi-frequency bioelectrical impedance compared to deuterium dilution for assessment of total body water. Nutrition \& Dietetics 2015 , 72:183-189.

26. Weyer C, Walford RL, Harper IT, et al.: Energy metabolism after 2 y of energy restriction: The biosphere 2 experiment. The American journal of clinical nutrition 2000, 72:946-953.

27. Karastergiou K, Smith SR, Greenberg AS, et al.: Sex differences in human adipose tissues - the biology of pear shape. Biol Sex Differ 2012, 3:13.

28. Liguori G and Medicine ACoS: Acsm's guidelines for exercise testing and prescription: Lippincott Williams \& Wilkins; 2020(Series Editor)

29. Mechelli F, Arendt-Nielsen L, Stokes M, et al.: Validity of ultrasound imaging versus magnetic resonance imaging for measuring anterior thigh muscle, subcutaneous fat, and fascia thickness. Methods Protoc 2019, 2.

30. Wagner DR, Thompson BJ, Anderson DA, et al:: A-mode and b-mode ultrasound measurement of fat thickness: A cadaver validation study. European journal of clinical nutrition 2019, 73:518-523.

31. Jackson AS, Pollock ML, and Ward A: Generalized equations for predicting body density of women. Medicine and science in sports and exercise 1980, 12:175-181.

32. Lixandrão ME, Ugrinowitsch C, Bottaro M, et al.: Vastus lateralis muscle cross-sectional area ultrasonography validity for image fitting in humans. J Strength Cond Res 2014, 28:3293-7.

33. Ahtiainen JP, Hoffren M, Hulmi JJ, et al.: Panoramic ultrasonography is a valid method to measure changes in skeletal muscle cross-sectional area. European journal of applied physiology 2010, 108:273-9.

34. Valera-Calero JA, Ojedo-Martín C, Fernández-de-Las-Peñas C, et al.: Reliability and validity of panoramic ultrasound imaging for evaluating muscular quality and morphology: A systematic review. Ultrasound in Medicine \& Biology 2020.

35. Franchi MV, Longo S, Mallinson J, et al.: Muscle thickness correlates to muscle cross-sectional area in the assessment of strength training-induced hypertrophy. Scandinavian journal of medicine \& science in sports 2018, 28:846-853.

36. Jeong J-R, Lee S-J, and Lee W-H: Reliability of rehabilitative ultrasound imaging for measuring the gluteus maximus muscle at rest and during contraction. Physical Therapy Rehabilitation Science 2017, 6:7-13. 
37. Casa DJ, Armstrong LE, Hillman SK, et al.: National athletic trainers' association position statement: Fluid replacement for athletes. J Athl Train 2000, 35:212-24.

38. : Exercise and fluid replacement. Medicine \& Science in Sports \& Exercise 2007, 39:377-390.

39. Sterling WM, Golden NH, Jacobson MS, et al.: Metabolic assessment of menstruating and nonmenstruating normal weight adolescents. International Journal of Eating Disorders 2009, 42:658663.

40. Kinney JM: Energy metabolism: Tissue determinants and cellular corollaries: raven Press; 1992(Series Editor)

41. Teixeira V, Voci SM, Mendes-Netto RS, et al.: The relative validity of a food record using the smartphone application myfitnesspal. Nutrition \& Dietetics 2018, 75:219-225.

42. Stone $M, O$ 'bryant $H$, Schilling $B$, et al.: Periodization: Effects of manipulating volume and intensity. Part 1. Strength \& Conditioning Journal 1999, 21:56.

43. Haff GG: Quantifying workloads in resistance training: A brief review. Strength Cond J 2010, 10:3140.

44. Organization WH: Who guidelines on drawing blood: Best practices in phlebotomy: World Health Organization; 2010(Series Editor)

45. Banasiak SJ, Wertheim EH, Koerner J, et al.: Test-retest reliability and internal consistency of a variety of measures of dietary restraint and body concerns in a sample of adolescent girls. International Journal of Eating Disorders 2001, 29:85-89.

46. Nakamoto $\mathrm{J}$ and Mason P: Quest diagnostics. The quest diagnostics manual endocrinology. San juan capistrano. CA: Quest Diagnostics 2012.

47. Zurlo F, Larson K, Bogardus C, et al.: Skeletal muscle metabolism is a major determinant of resting energy expenditure. The Journal of clinical investigation 1990, 86:1423-7.

48. Sundgot-Borgen J, Meyer NL, Lohman TG, et al.: How to minimise the health risks to athletes who compete in weight-sensitive sports review and position statement on behalf of the ad hoc research working group on body composition, health and performance, under the auspices of the ioc medical commission. British Journal of Sports Medicine 2013, 47:1012-1022.

49. STAND P: The female athlete triad. Med Sci Sports Exerc 2007, 39:1867-82.

50. Bryner RW, Ullrich IH, Sauers J, et al.: Effects of resistance vs. Aerobic training combined with an 800 calorie liquid diet on lean body mass and resting metabolic rate. Journal of the American College of Nutrition 1999, 18:115-121.

51. Ardavani A, Aziz H, Smith K, et al.: The effects of very low energy diets and low energy diets with exercise training on skeletal muscle mass: A narrative review. Advances in Therapy 2020:1-15.

52. Mettler S, Mitchell N, and Tipton KD: Increased protein intake reduces lean body mass loss during weight loss in athletes. Medicine \& Science in Sports \& Exercise 2010, 42:326-337.

53. Helms ER, Zinn C, Rowlands DS, et al.: A systematic review of dietary protein during caloric restriction in resistance trained lean athletes: A case for higher intakes. International journal of sport nutrition 
and exercise metabolism 2014, 24:127-138.

54. Neto WK, Soares EG, Vieira TL, et al.: Gluteus maximus activation during common strength and hypertrophy exercises: A systematic review. J Sports Sci Med 2020, 19:195-203.

55. Toomey CM, McCormack WG, and Jakeman P: The effect of hydration status on the measurement of lean tissue mass by dual-energy x-ray absorptiometry. European journal of applied physiology 2017, 117:567-574.

56. Barreira $T$ and Tseh $\mathrm{W}$ : The effects of acute water ingestion on body composition analyses via dualenergy $x$-ray absorptiometry. Clinical Nutrition 2020.

57. Tinsley GM, Morales E, Forsse JS, et al.: Impact of acute dietary manipulations on dxa and bia body composition estimates. Medicine and science in sports and exercise 2017, 49:823-832.

58. Roberts BM, Helms ER, Trexler ET, et al.: Nutritional recommendations for physique athletes. J Hum Kinet 2020, 71:79-108.

59. Hawley JA, Schabort EJ, Noakes TD, et al.: Carbohydrate-loading and exercise performance. An update. Sports medicine 1997, 24:73-81.

60. Ahola R, Korpelainen R, Vainionpää A, et al.: Time-course of exercise and its association with 12month bone changes. BMC musculoskeletal disorders 2009, 10:138.

61. Shirreffs S: Markers of hydration status. European journal of clinical nutrition 2003, 57:S6-S9.

62. Chappell AJ and Simper TN: Nutritional peak week and competition day strategies of competitive natural bodybuilders. Sports 2018, 6:126.

63. Mayr FB, Domanovits H, and Laggner AN: Hypokalemic paralysis in a professional bodybuilder. The American Journal of Emergency Medicine 2012, 30:1324. e5-1324. e8.

64. Heshka S, Yang M-U, Wang J, et al.: Weight loss and change in resting metabolic rate. The American journal of clinical nutrition 1990, 52:981-986.

65. Yavuz S, Salgado Nunez del Prado S, and Celi FS: Thyroid hormone action and energy expenditure. Journal of the Endocrine Society 2019, 3:1345-1356.

66. Van Pelt RE, Dinneno FA, Seals DR, et al.: Age-related decline in rmr in physically active men: Relation to exercise volume and energy intake. American Journal of Physiology-Endocrinology and Metabolism 2001, 281:E633-E639.

67. Byrne NM, Weinsier RL, Hunter GR, et al.: Influence of distribution of lean body mass on resting metabolic rate after weight loss and weight regain: Comparison of responses in white and black women. The American journal of clinical nutrition 2003, 77:1368-1373.

68. Gold EB: The timing of the age at which natural menopause occurs. Obstet Gynecol Clin North Am 2011, 38:425-40.

69. Hale GE, Robertson DM, and Burger HG: The perimenopausal woman: Endocrinology and management. The Journal of steroid biochemistry and molecular biology 2014, 142:121-131.

70. Loucks A, Verdun M, and Heath E: Low energy availability, not stress of exercise, alters Ih pulsatility in exercising women. Journal of applied physiology 1998, 84:37-46. 
71. Herbison AE: Control of puberty onset and fertility by gonadotropin-releasing hormone neurons. Nature Reviews Endocrinology 2016, 12:452.

72. Klok MD, Jakobsdottir S, and Drent M: The role of leptin and ghrelin in the regulation of food intake and body weight in humans: A review. Obesity reviews 2007, 8:21-34.

73. Tena-Sempere M: Roles of ghrelin and leptin in the control of reproductive function. Neuroendocrinology 2007, 86:229-241.

74. Ibrahim Abdalla MM: Ghrelin - physiological functions and regulation. Eur Endocrinol 2015, 11:90-95.

75. Stubbs BJ, Cox PJ, Evans RD, et al.: A ketone ester drink lowers human ghrelin and appetite. Obesity 2018, 26:269-273.

76. Korek E, Krauss H, Gibas-Dorna M, et al.: Fasting and postprandial levels of ghrelin, leptin and insulin in lean, obese and anorexic subjects. Przeglad gastroenterologiczny 2013, 8:383.

77. Blatnik $\mathrm{M}$ and Soderstrom $\mathrm{Cl}$ : A practical guide for the stabilization of acylghrelin in human blood collections. Clinical endocrinology 2011, 74:325-331.

78. Narici MV, Hoppeler H, Kayser B, et al.: Human quadriceps cross-sectional area, torque and neural activation during 6 months strength training. Acta Physiol Scand 1996, 157:175-86.

79. Loucks $A B$ and Thuma JR: Luteinizing hormone pulsatility is disrupted at a threshold of energy availability in regularly menstruating women. The Journal of Clinical Endocrinology \& Metabolism 2003, 88:297-311.

\section{Tables}

Due to technical limitations, table 4, 5, 6 is only available as a download in the Supplemental Files section.

Table 7 not available with this version

\section{Figures}



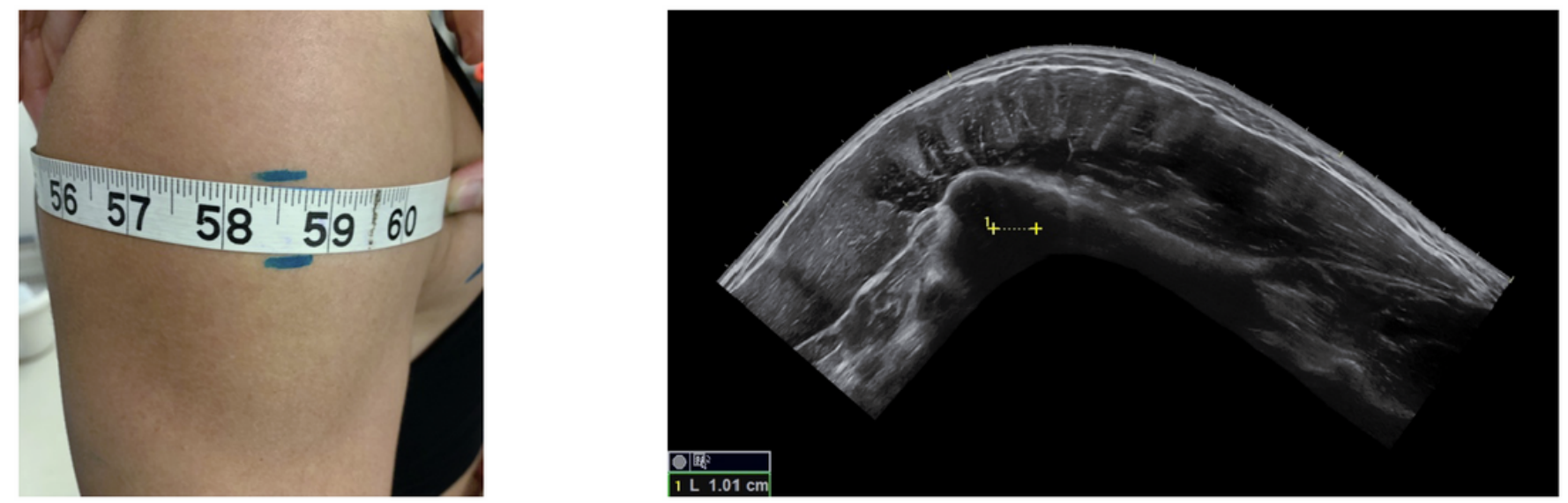

\section{Figure 1}

The image on the left portrays the area of interest where the largest semi-circumference of the right deltoid was located and marked. Two lines were made above and below the tape measure. The transducer followed a path from the pectoralis major and the anterior deltoid interact to the furthest point posterior to capture the most medial portion of the posterior deltoid. The image on the right is the Delt 

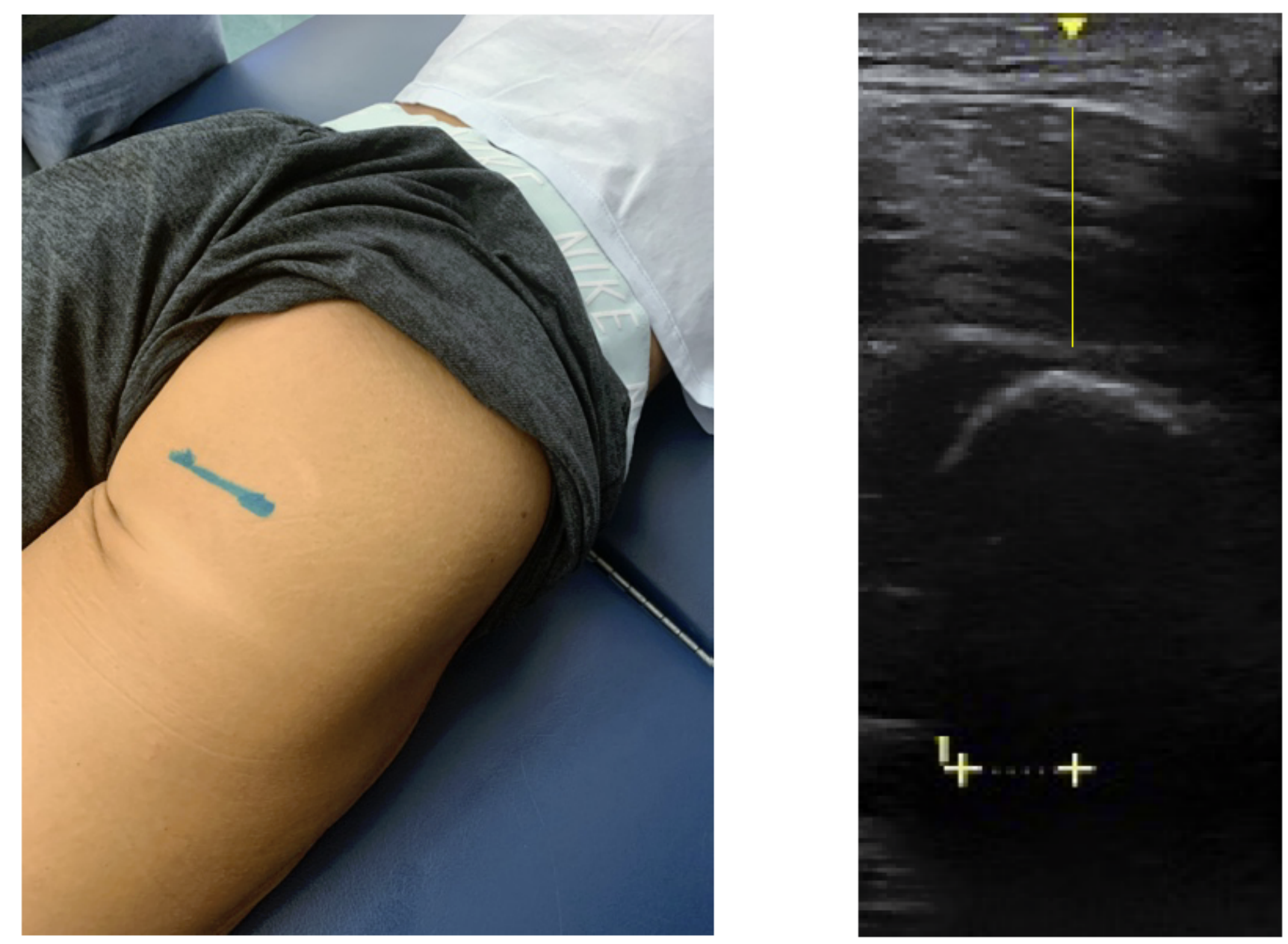

\section{Figure 2}

The image of the left shows the marked location of the ischial tuberosity (IT). The image on the right shows the US measurement taken to assess GMMT. 

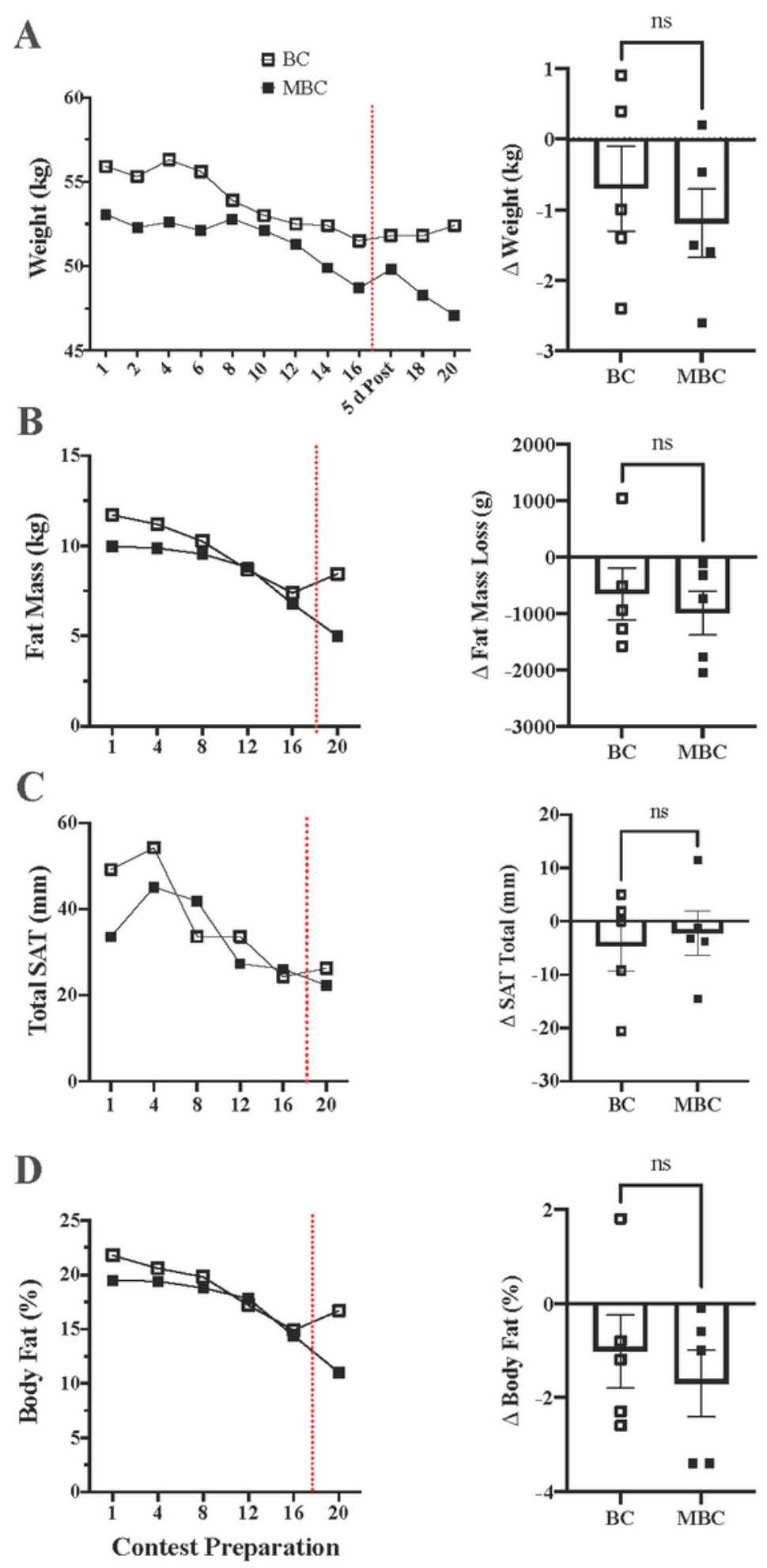

\section{Figure 3}

The time course analysis of 20-week contest preparation of A) Weight, B) Fat mass, C) Total subcutaneous adipose tissue (SAT), and D) Body fat \%. Each time course analysis was accompanied with 4-week interval $\triangle$ change (mean \pm SEM). No significant differences were found in mean change between $\mathrm{BC}$ and $\mathrm{MBC}$. The dashed red line denotes the competition. 
ns
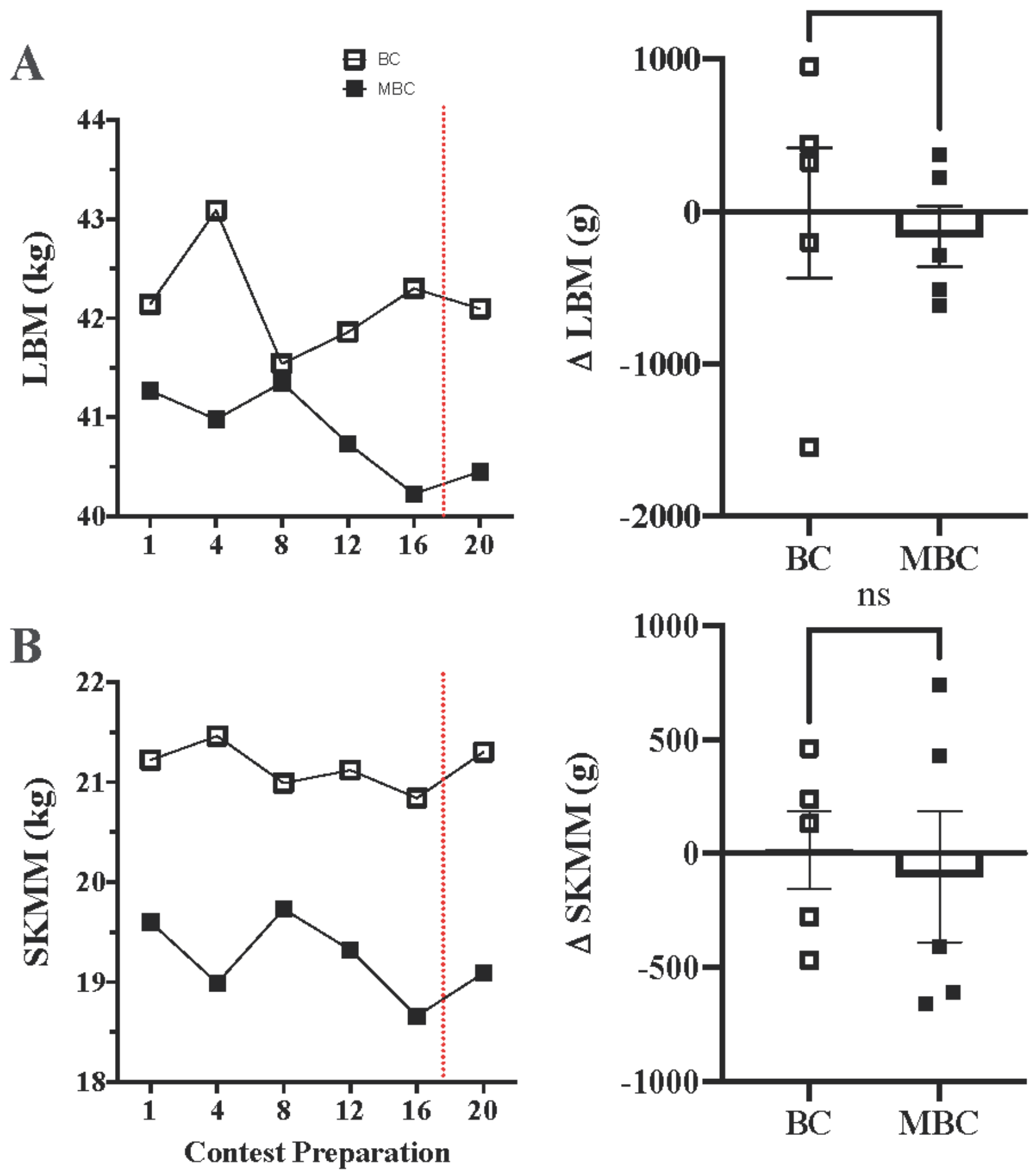

Figure 4

The time course analysis of 20-week contest preparation of A) lean body mass (LBM) and B) estimated skeletal muscle mass (SKMM). Each time course analysis was accompanied with 4-week interval $\triangle$ change (mean \pm SEM). No significant differences were found in mean change between BC and MBC. The dashed red line denotes the competition. 

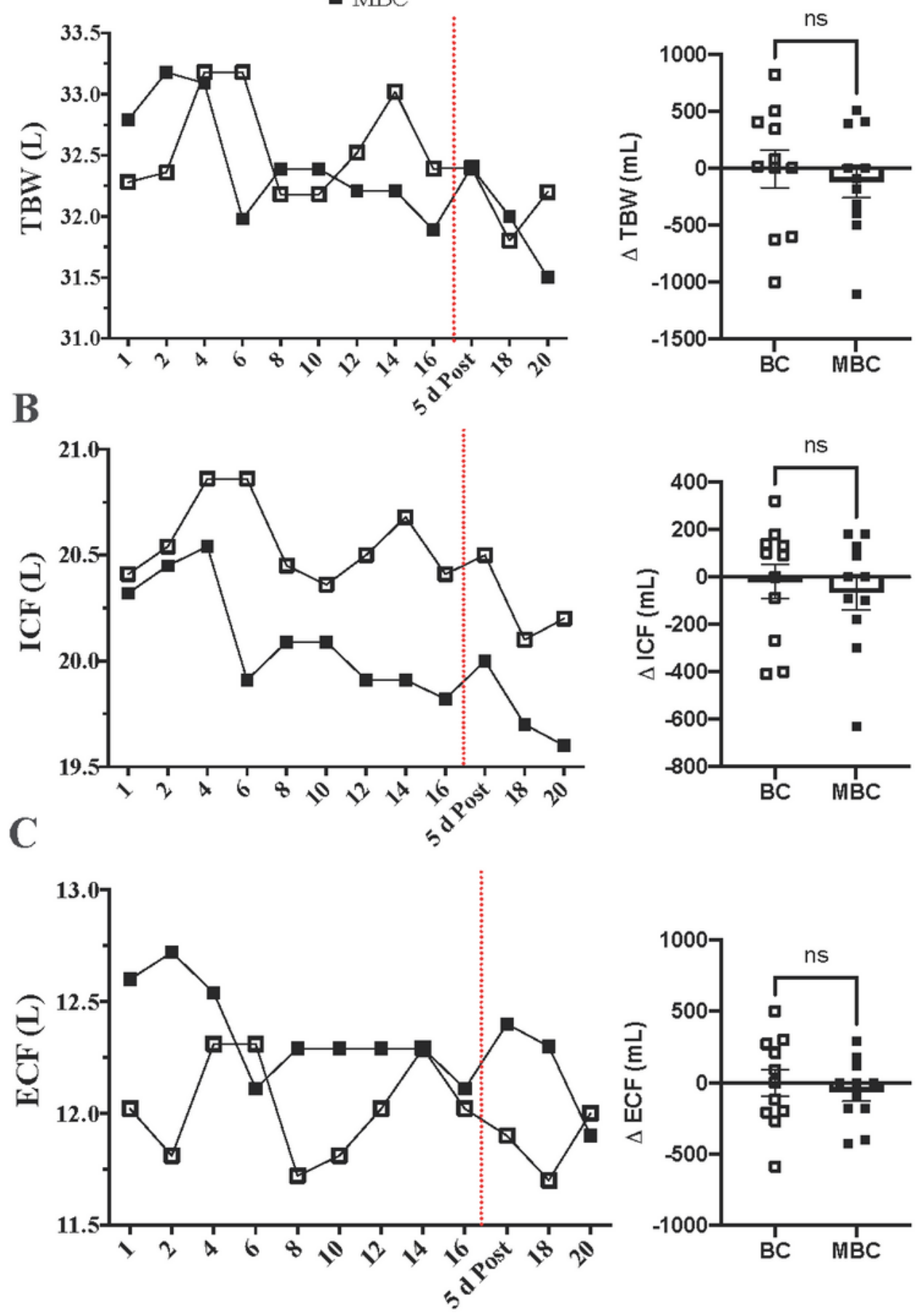

\section{Contest Preparation}

\section{Figure 5}

The time course analysis of 20-week contest preparation of A) total body water (TBW), B) intracellular fluid (ICF), and C) extracellular fluid (ECF). Each time course analysis was accompanied with bi-weekly interval $\triangle$ change (mean $\pm \mathrm{SEM}$ ). No significant differences were found in mean change between $\mathrm{BC}$ and MBC. The dashed red line denotes the competition. 

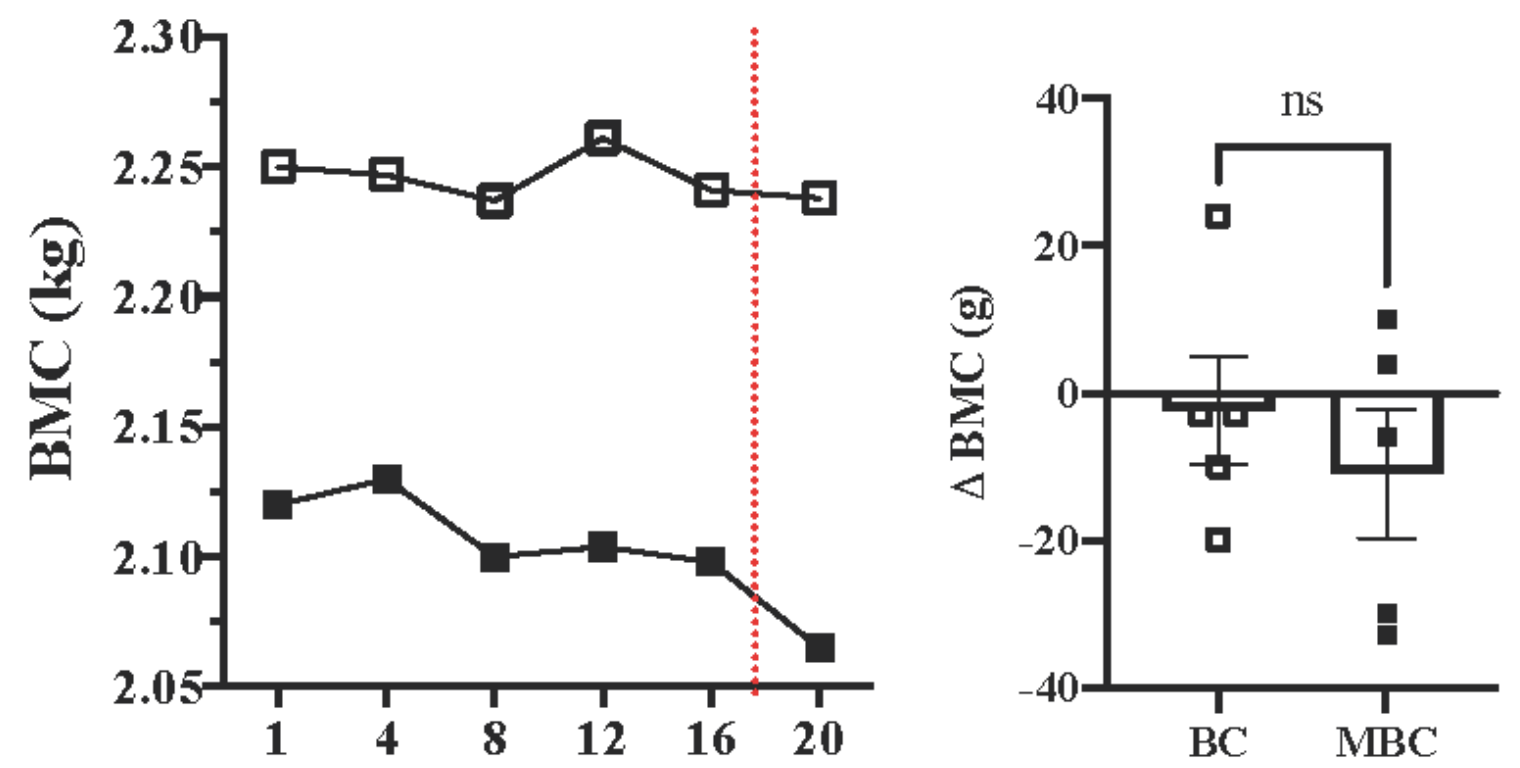

B

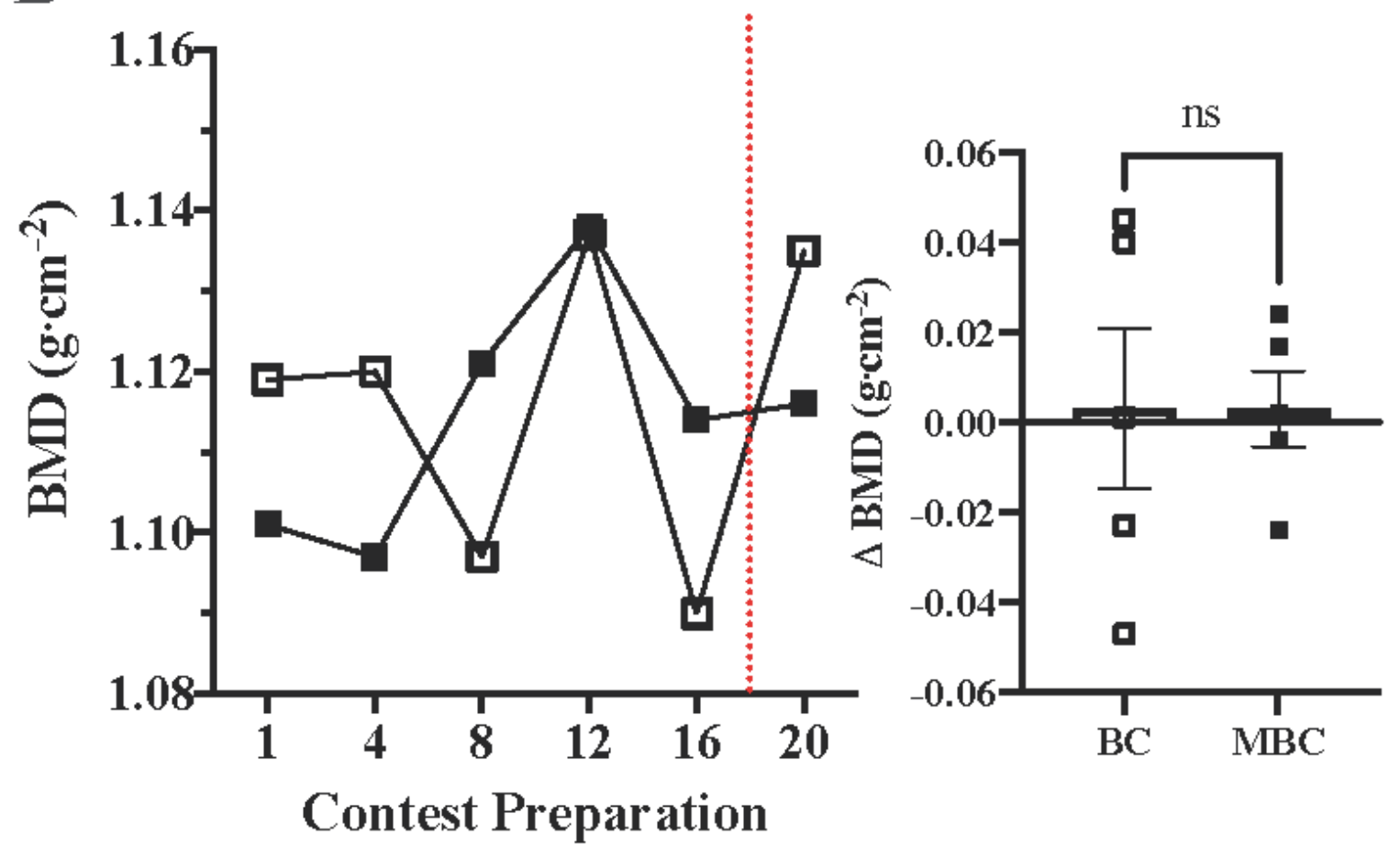

Figure 6

The time course analysis of 20-week contest preparation of A) bone mineral content (BMC) and B) bone mineral density (BMD). Each time course analysis was accompanied with 4-week interval $\triangle$ change (mean \pm SEM). No significant differences were found in mean change between BC and MBC. The dashed red line denotes the competition. 


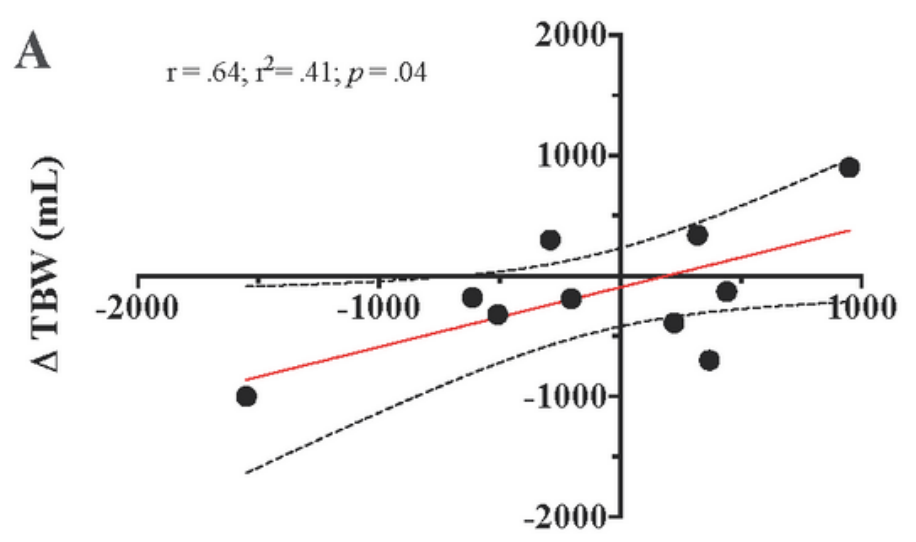

$\triangle$ LBM (g)

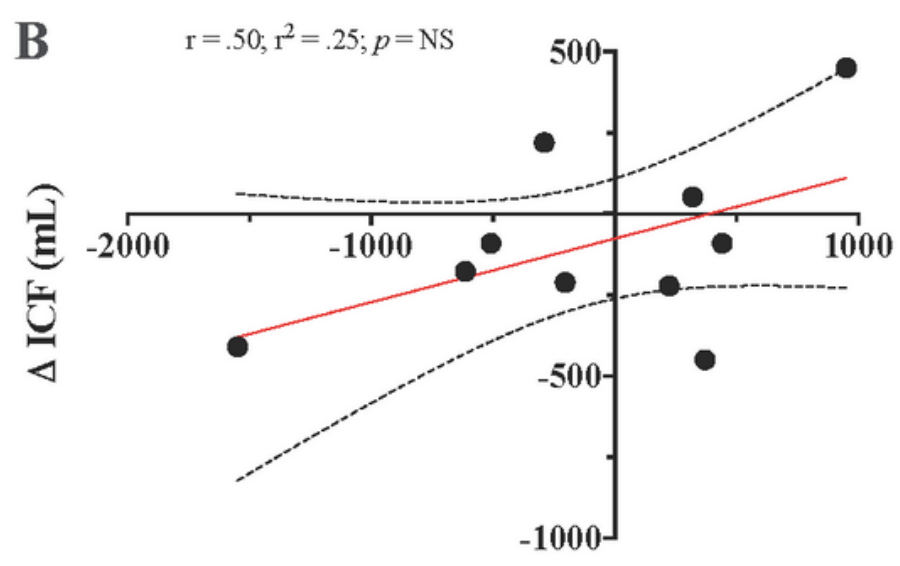

$\triangle \operatorname{LBM}(\mathbf{g})$

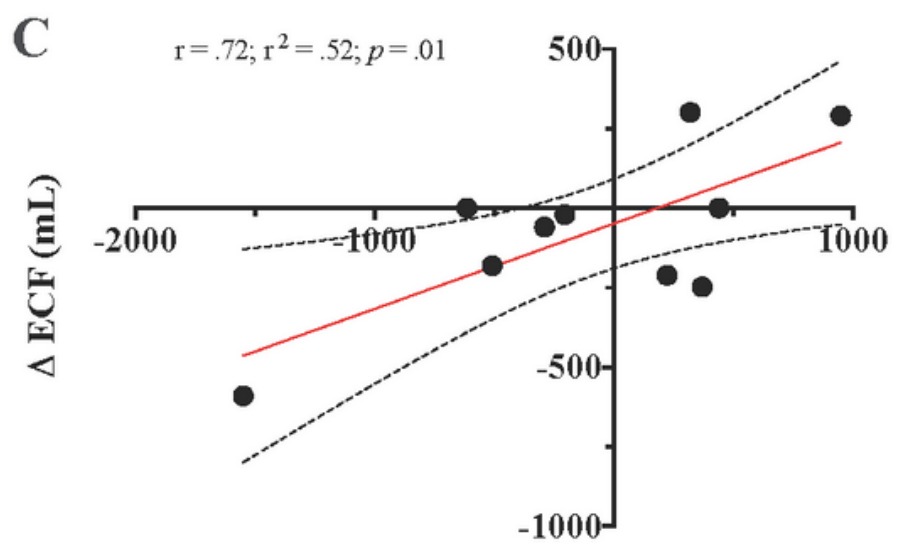

$\triangle$ LBM (g)

\section{Figure 7}

Assessing correlations between A) $\Delta$ total body water (TBW) and $\Delta$ lean body mass (LBM); B) $\Delta$ intracellular fluid (ICF) and $\triangle \mathrm{LBM} ; \mathrm{C}$ ) $\Delta$ extracellular fluid (ECF) and $\triangle \mathrm{LBM}$ significant relationships were found between $\Delta$ TBW and $\Delta$ LBM $(p=.04)$, and $\triangle$ ECF and $\Delta$ LBM $(p=.01)$. NS was found for $\Delta$ ICF and $\triangle$ LBM. 


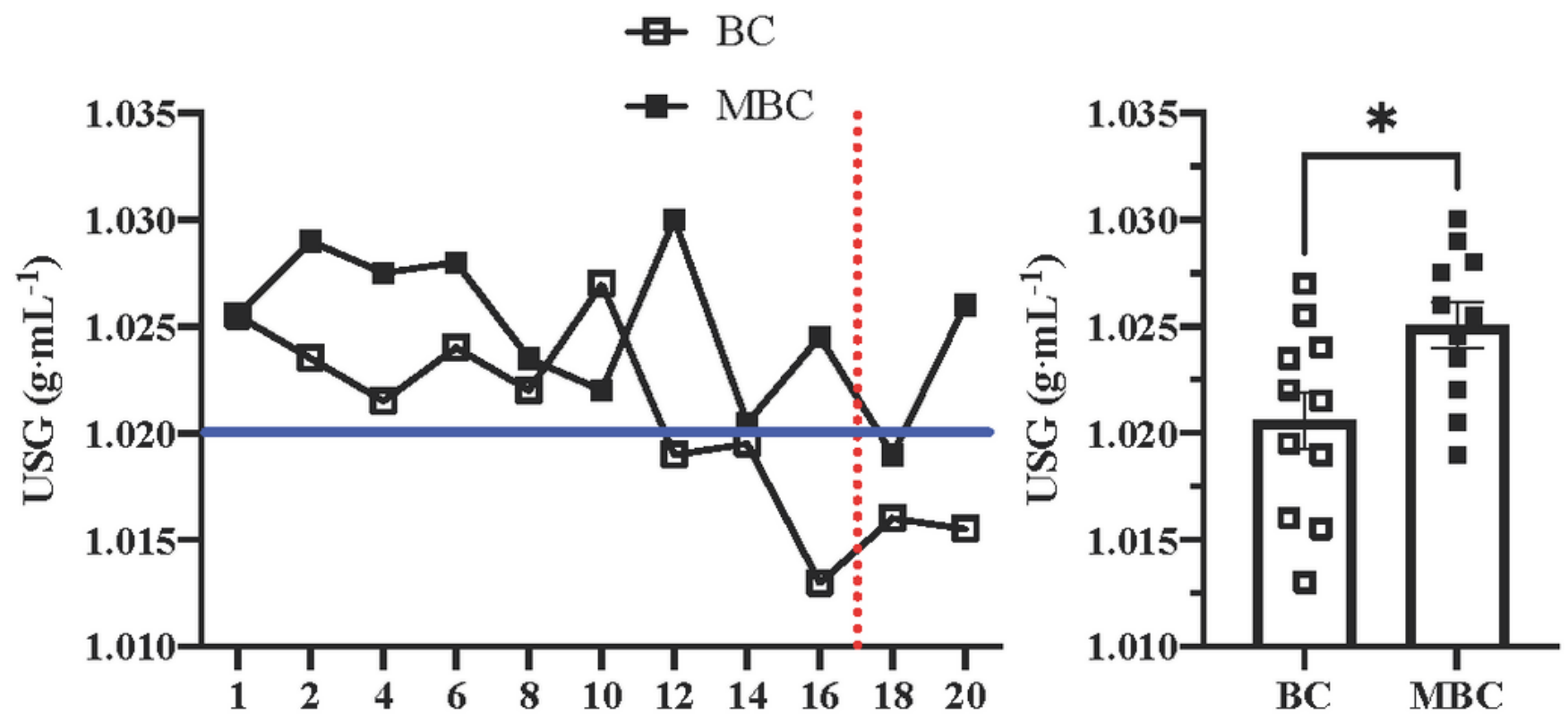

Contest Preparation

B
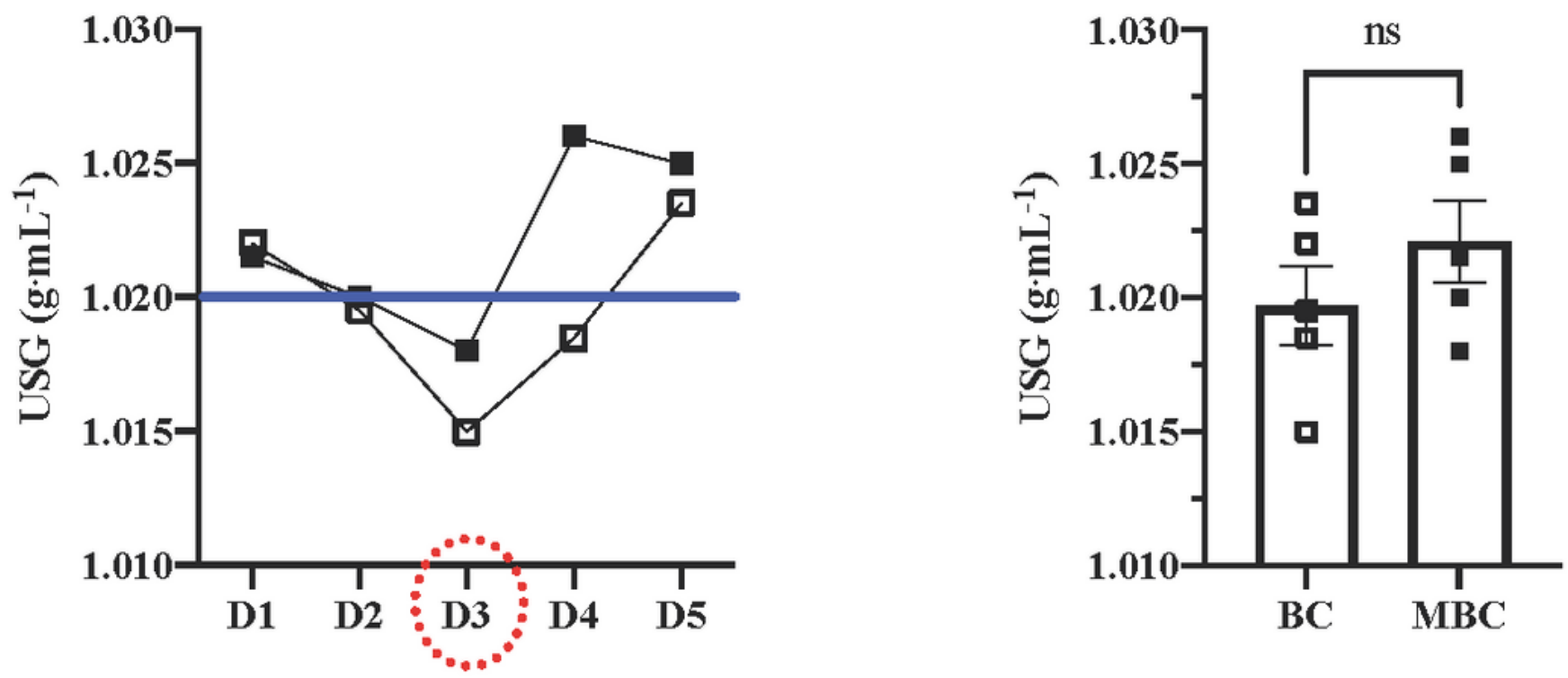

Competition Week

Figure 8

The time course analysis of 20-week contest preparation of hydration status assessed with urine specific gravity (USG) for A) Contest Preparation (20 weeks). The time course analysis was accompanied with assessment of mean average (mean \pm SEM) over 20-weeks. A significant mean USG difference $(* p=.01)$ was found between BC (1.021 $\pm .001 ; 95 \% \mathrm{Cl}$ : 1.018-1.024) and MBC (1.025 $\pm .001 ; 95 \% \mathrm{Cl}$ : 1.023-1.027). (B) Competition Week time course analysis assessed with USG over 5 d (D1-D5). NS was found between 
$B C$ and MBC. The dashed red line and circle denotes the competition. The solid blue line denotes the euhydration threshold $(1.020 \mathrm{~g} \cdot \mathrm{mL}-1$

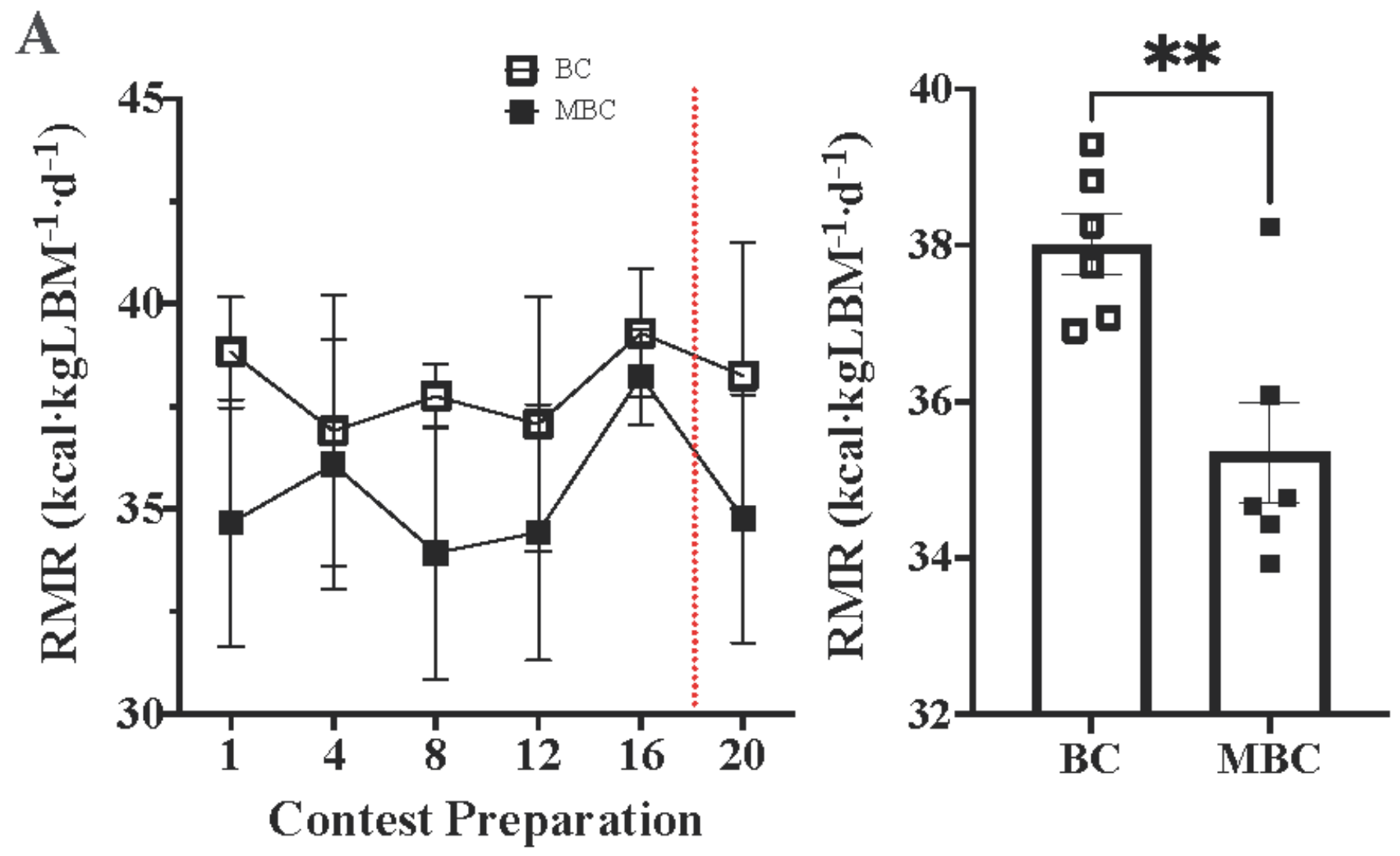

Figure 9

Exploratory assessment of the time course of RMR during 20-week contest preparation with accompanying mean average (mean \pm SEM) comparison between $B C$ and MBC. A) No RMR differences were found between $B C$ and MBC at any time after normalizing to $\mathrm{kcal} \cdot \mathrm{kgLBM}-1 \cdot \mathrm{d}-1$. There was a difference $(* * \mathrm{p}=.005)$ found between BC (38.01 $\pm 0.38 \mathrm{kcal} \cdot \mathrm{kgLBM}-1 \cdot \mathrm{d}-1 ; 95 \% \mathrm{Cl}: 37.01-39.01)$ and MBC (35.35 $\pm 0.64 \mathrm{kcal} \cdot \mathrm{kgLBM}-1 \cdot \mathrm{d}-1 ; 95 \% \mathrm{Cl}$ : 33.69-37.01). The red dashed line denotes the competition. 
A $\quad \begin{array}{r}\mathrm{BC} \\ \quad \mathrm{MBC}\end{array}$
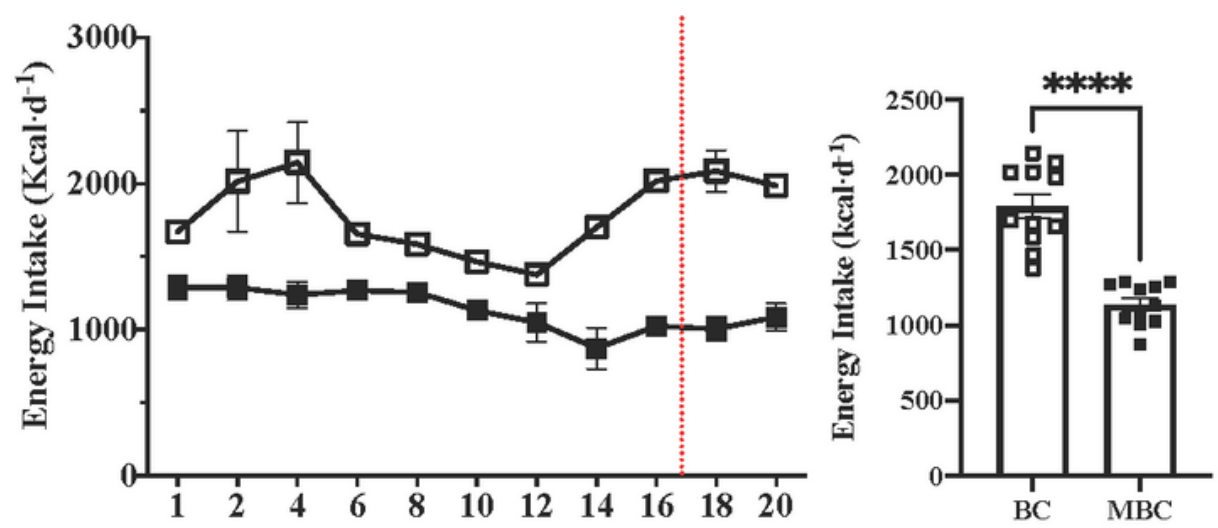

B

\section{Figure 10}

The time course analysis of 20-week contest preparation for energy intake from the self-reported 4-day dietary recall with accompanying mean average (mean \pm SEM) comparison between $B C$ and $M B C$. A) A

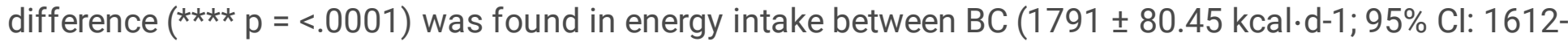
1912) and MBC (1137 $\pm 42.35 \mathrm{kcal} \cdot \mathrm{d}-1 ; 95 \% \mathrm{Cl}$ : 1043-1232). B) A difference ( $* \star \star \star ~ p=<.0001)$ was found in energy intake between BC (34.18 $\pm 1.85 \mathrm{kcal} \cdot \mathrm{kg}-1 \cdot \mathrm{d}-1 ; 95 \% \mathrm{Cl}: 30.04-38.31)$ and MBC $(22.25 \pm 0.68$ 
$\mathrm{kcal} \cdot \mathrm{kg}-1 \cdot \mathrm{d}-1 ; 95 \% \mathrm{Cl}: 20.71-23.78)$. C) A difference (** $\mathrm{p}=.001)$ was found in energy intake between BC $(43.20 \pm 3.24 \mathrm{kcal} \cdot \mathrm{kgLBM}-1 \cdot \mathrm{d}-1 ; 95 \% \mathrm{Cl}: 25.26-31.01)$. The red dashed lines denote competition.

A
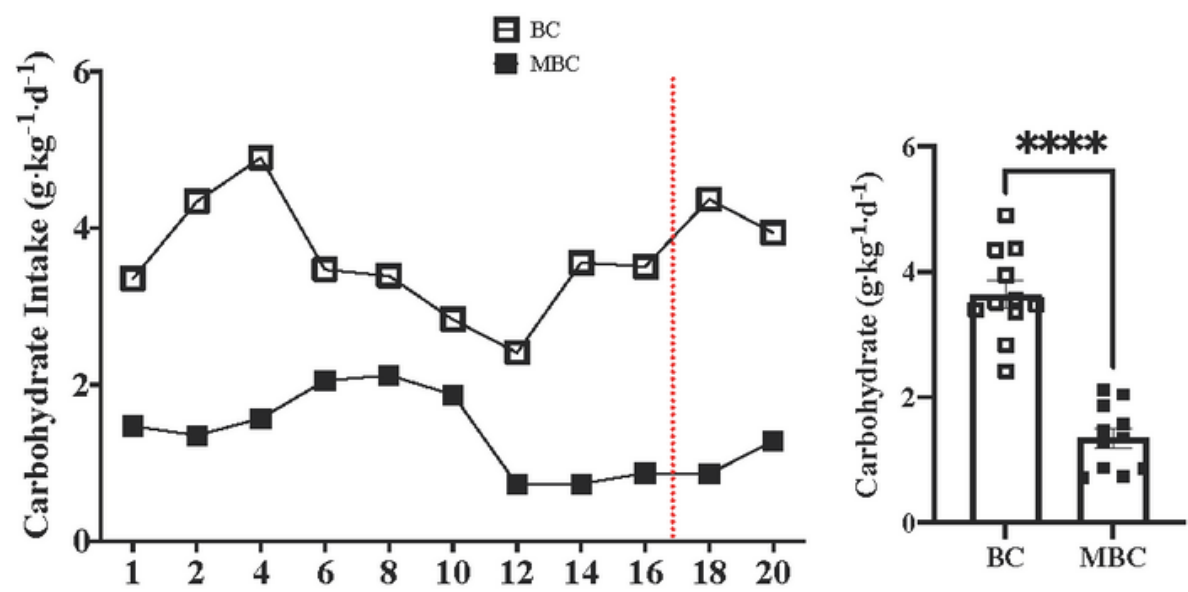

B
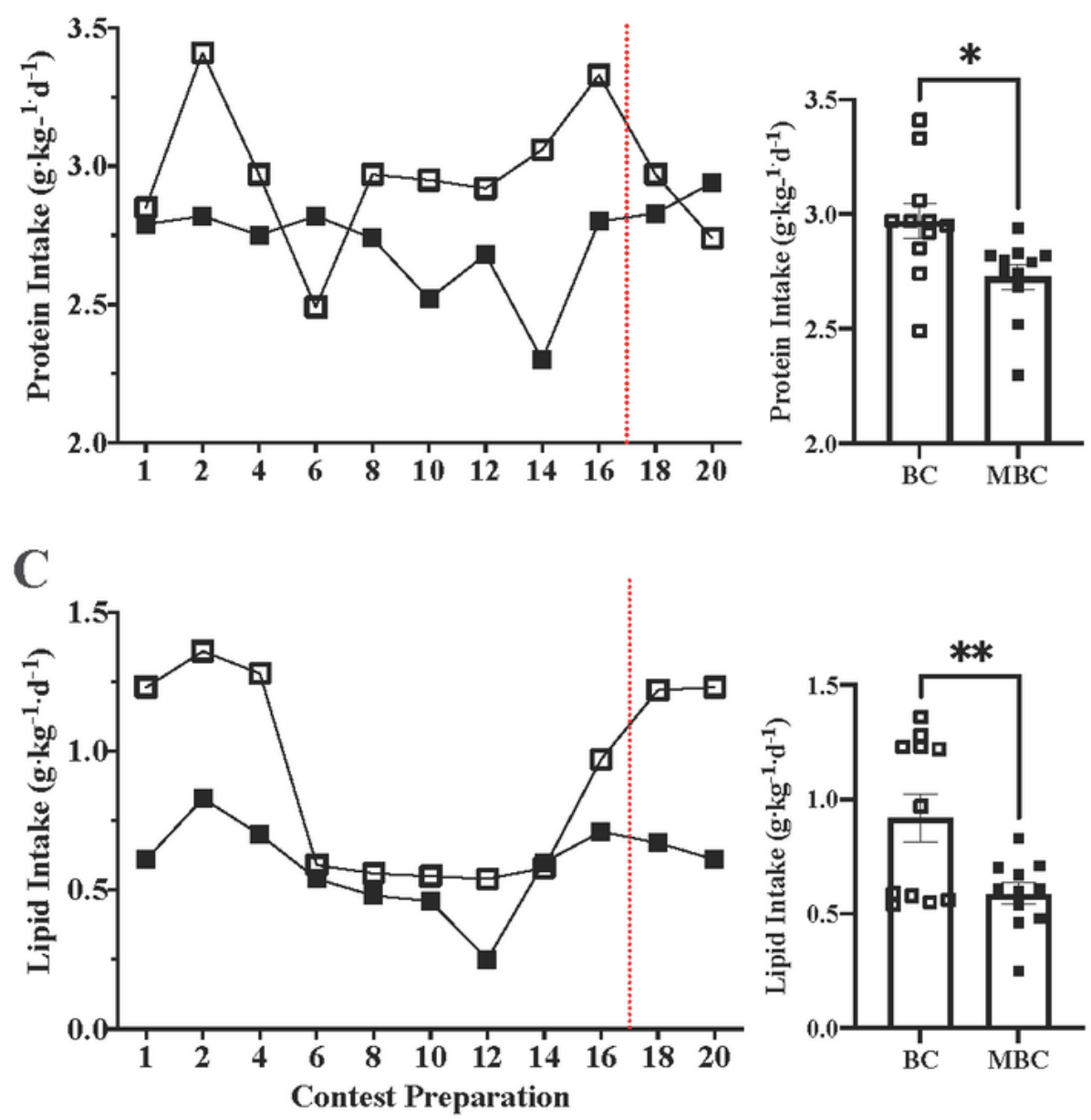

Figure 11

The time course analysis of the 20-week contest preparation macronutrient intake from the self-reported 4-day dietary recall with accompanying mean average (mean $\pm \mathrm{SEM}$ ) comparison between $\mathrm{BC}$ and MBC. A) A difference $(* \star \star \star ~ p=<.0001)$ was found in carbohydrate intake between BC $(3.64 \pm 0.21 \mathrm{~g} \cdot \mathrm{kg}-1 \cdot \mathrm{d}-1$; 
$95 \% \mathrm{Cl}: 3.16-4.12)$ and MBC (1.354 $\pm 0.15 \mathrm{~g} \cdot \mathrm{kg}-1 \cdot \mathrm{d}-1 ; 95 \% \mathrm{Cl}: 1.00-1.70)$. B) A difference $(* \mathrm{p}=0.0163)$ was found in protein intake between BC (2.96 $\pm 0.07 \mathrm{~g} \cdot \mathrm{kg}-1 \cdot \mathrm{d}-1 ; 95 \% \mathrm{Cl}: 2.80-3.13)$ and MBC $(2.72 \pm 0.05$ $\mathrm{g} \cdot \mathrm{kg}-1 \cdot \mathrm{d}-1 ; 95 \% \mathrm{Cl}: 2.60-2.84)$. C) A difference (** $\mathrm{p}=0.009)$ was found lipid intake between BC (0.91 \pm $0.10 \mathrm{~g} \cdot \mathrm{kg}-1 \cdot \mathrm{d}-1 ; 95 \% \mathrm{Cl}: 0.6822-1.156)$ and MBC $(0.58 \pm 0.04 \mathrm{~g} \cdot \mathrm{kg}-1 \cdot \mathrm{d}-1 ; 95 \% \mathrm{Cl}: 0.4835-0.6910)$. The red dashed line denotes competition.
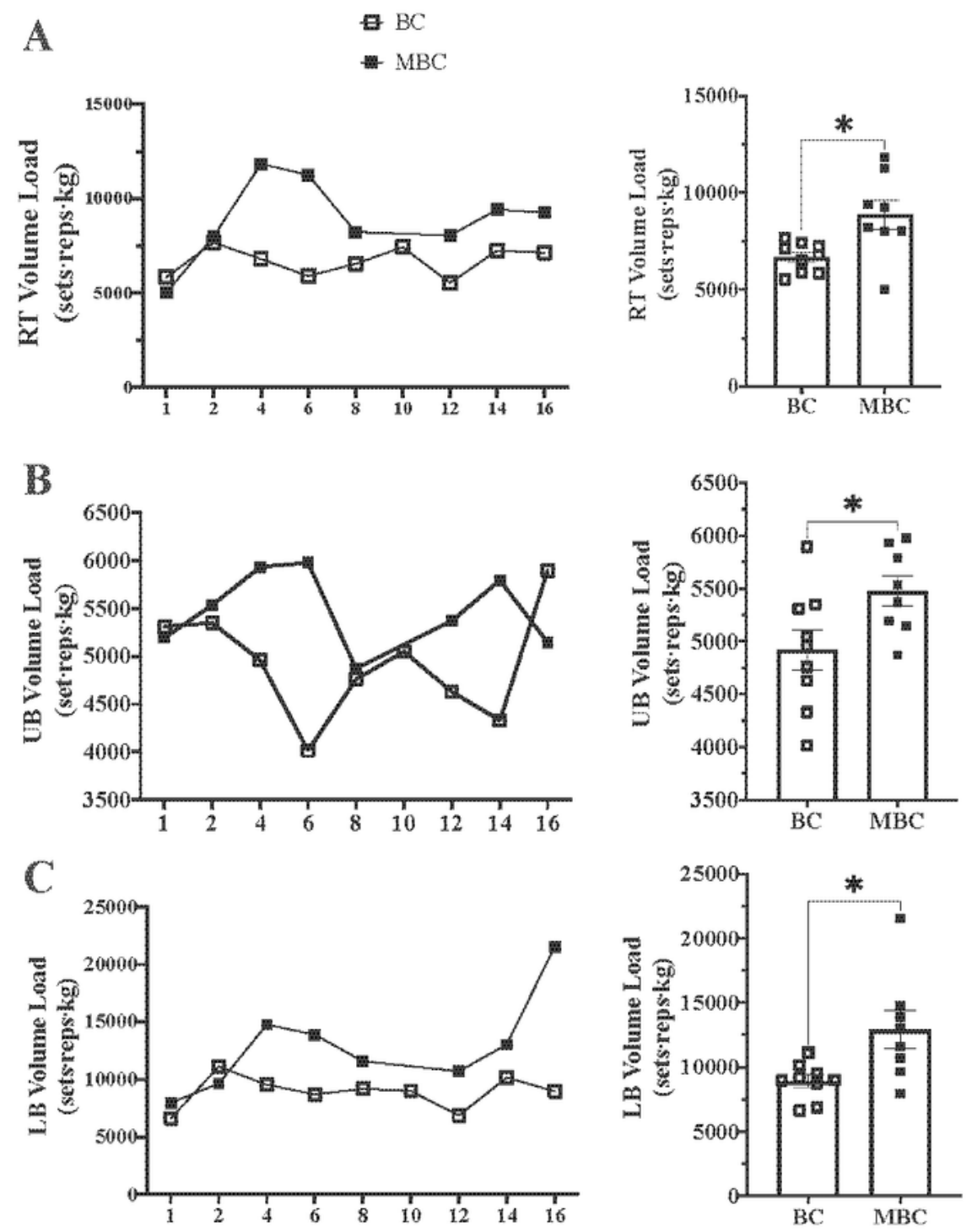

D
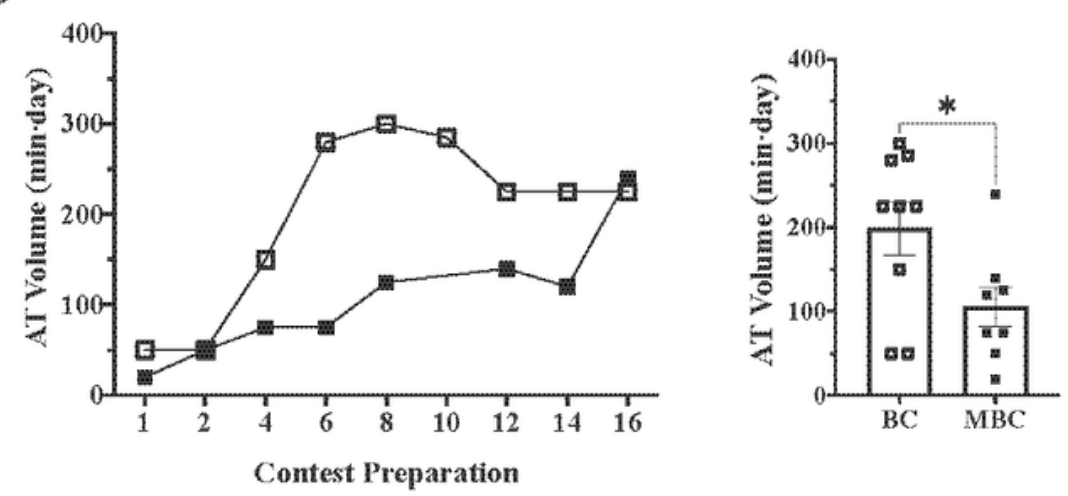

Figure 12 
Time course analysis during 16-week pre-contest preparation prior to competition of self-reported resistance training (RT) volume load (sets $\square$ reps $\square \mathrm{kg}$ ) and aerobic training (AT) volume ( $\min \square \mathrm{d}$ ) with accompanying mean average (mean \pm SEM) comparison between $B C$ and MBC. A) A difference was found $(* p=.01)$ in the mean total RT volume load between BC $(6669 \pm 256.1 ; 95 \% \mathrm{Cl}$ : 6078-7,259) and MBC ( $8879 \pm 752.0 ; 95 \%$ Cl: 7100-10657). B) A difference ( $\left.{ }^{*} p=.03\right)$ was found between mean upper body (UB) volume load between BC (4921 \pm 189.6 ; $95 \% \mathrm{Cl}$ : 4484-5358) and MBC (5477 $\pm 141.9 ; 95 \% \mathrm{Cl}$ : 5142-5813). C) A difference ( $p=.01$ ) was found between mean lower body (LB) volume load between BC $(8909 \pm 476.9 ; 95 \% \mathrm{Cl}: 7809-10009)$ and $\mathrm{MBC}(12987 \pm 1470 ; 95 \% \mathrm{Cl}: 9421-16373)$. D) A difference $(* \mathrm{p}=$ .03) was found in mean AT volume between BC (198.9 $\pm 31.84 ; 95 \% \mathrm{Cl}: 125.5-272.3)$ and MBC (105.6 \pm 23.95; 95\% Cl: 48.99-162.3). 
A
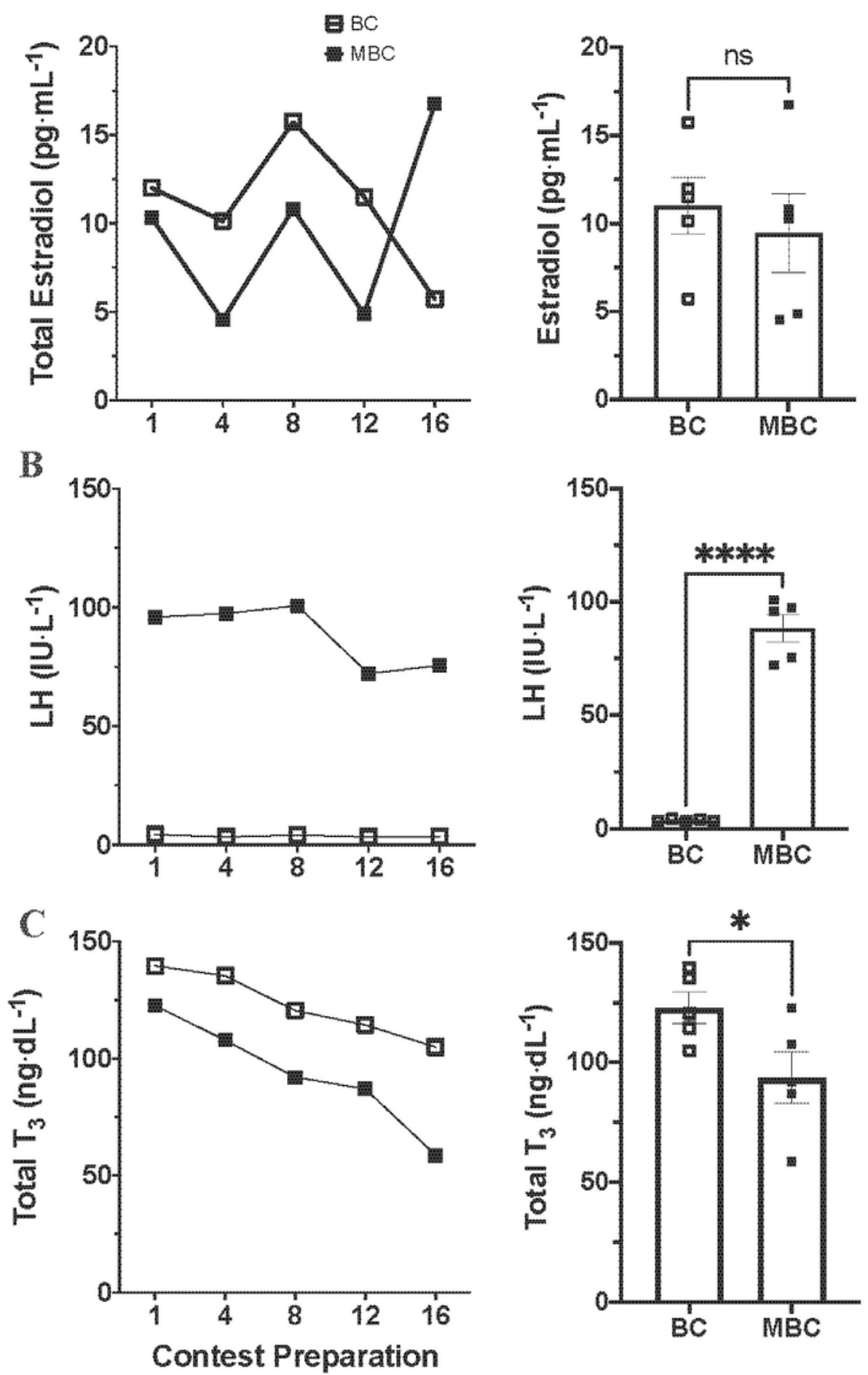

Figure 13

Time course analysis during 16-week pre-contest preparation of reproductive and metabolic hormones with accompanying mean differences (mean \pm SEM). A) No mean difference (ns) in Estradiol concentration was found between BC and MBC. B) A difference ( $\left.{ }^{* \star \star} p=<.0001\right)$ was found in mean luteinizing hormone $(\mathrm{LH})$ concentration between $\mathrm{BC}(3.66 \pm 0.23 \mathrm{IU} \cdot \mathrm{L}-1 ; 95 \% \mathrm{Cl}: 3.00-4.31)$ and MBC (88.34 $\pm 6.01 \mathrm{IU} \cdot \mathrm{L}-1 ; 95 \% \mathrm{Cl}: 71.65-105.0)$. C) A difference (* $p=.04$ ) was found in triiodothyronine (T3) 
between BC (122.9 $\pm 6.46 \mathrm{ng} \cdot \mathrm{dL}-1 ; 95 \% \mathrm{Cl}: 105.0-140.9)$ and MBC (93.64 $\pm 10.75 \mathrm{ng} \cdot \mathrm{dL}-1 ; 95 \% \mathrm{Cl}$ : 63.78123.5).
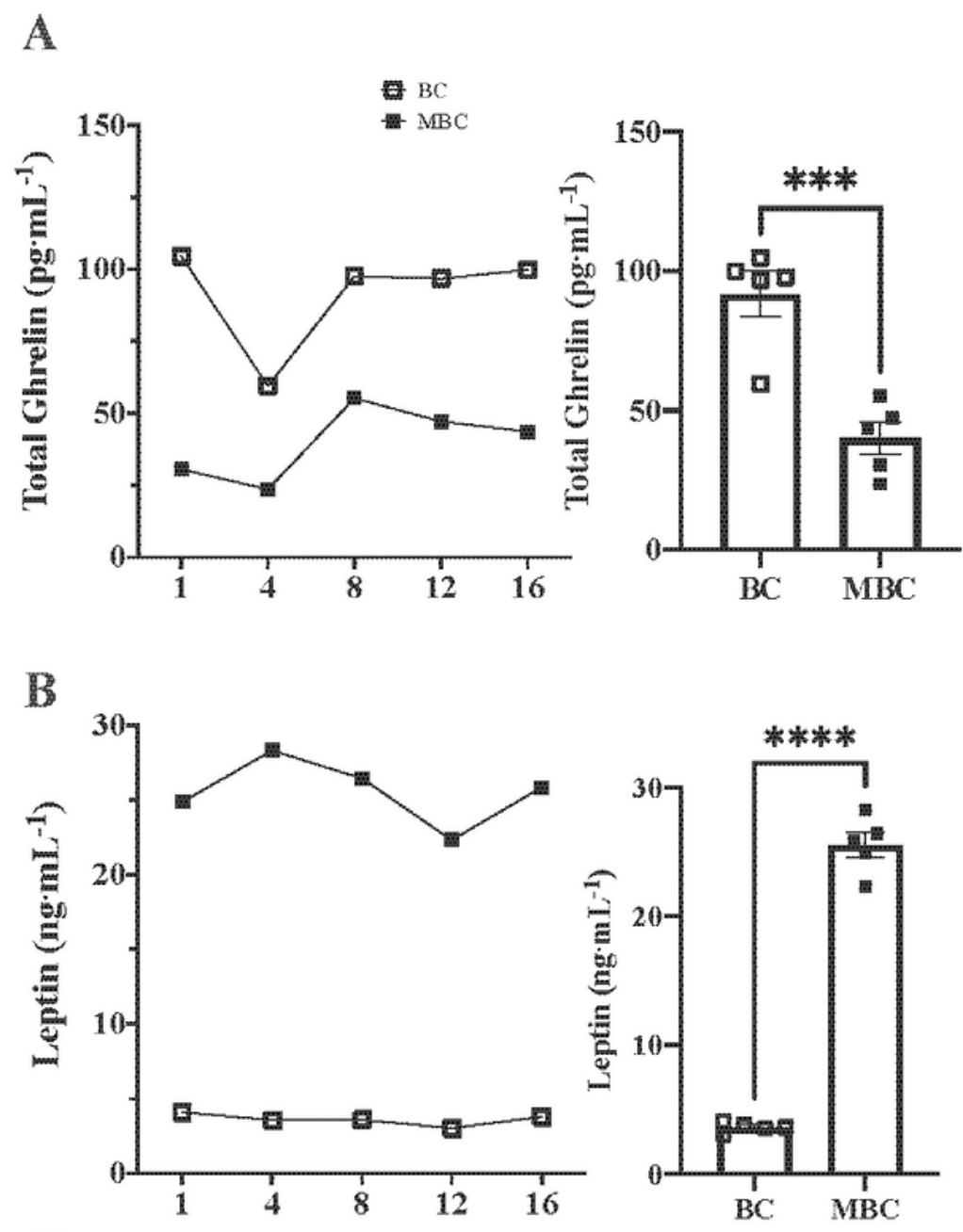

$\mathrm{C}$

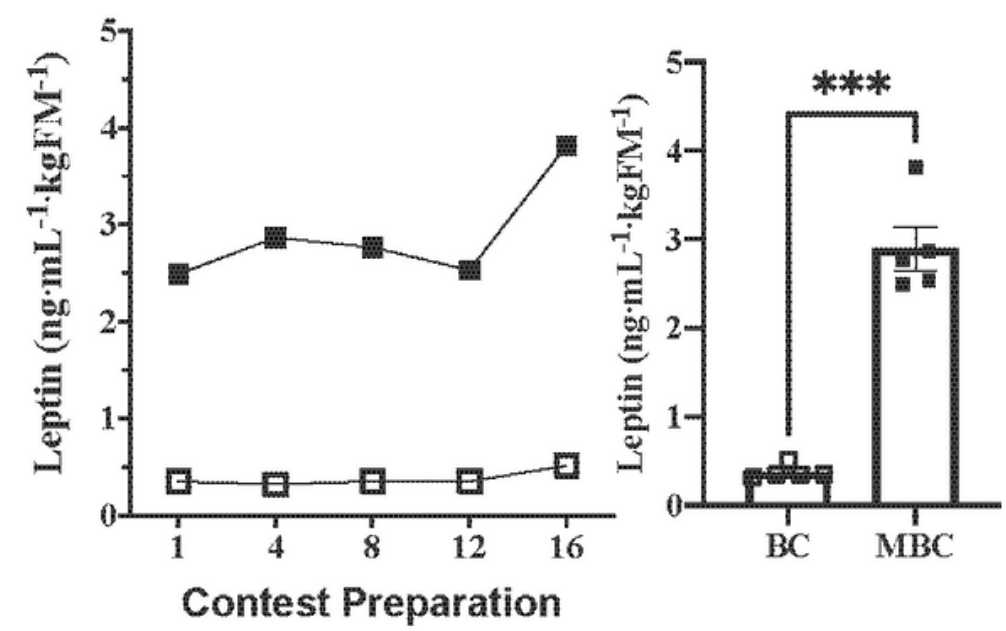

Figure 14

Time course analysis during 16-week pre-contest preparation of energy balance hormones with accompanying mean differences (mean \pm SEM). A) A mean difference $(* \star \star p=.008)$ in ghrelin concentration was found between BC (91.63 $\pm 8.14 \mathrm{pg} \cdot \mathrm{mL}-1 ; 95 \% \mathrm{Cl}: 69.01-114.2)$ and MBC (40.05 \pm 5.71 
$\mathrm{pg} \cdot \mathrm{mL}-1 ; 95 \% \mathrm{Cl}: 24.18-55.92)$. B) A difference $(\star \star \star \star ~ p=.005)$ was found in mean leptin concentration between BC (3.61 $\pm 0.17 \mathrm{ng} \cdot \mathrm{mL}-1 ; 95 \% \mathrm{Cl}: 3.13-4.09)$ and MBC $(25.55 \pm 0.98 \mathrm{ng} \cdot \mathrm{mL}-1 ; 95 \% \mathrm{Cl}: 22.81-$ 28.28). C) A difference ( $\left.{ }^{\star \star \star} p=.0004\right)$ in leptin concentration normalized by $\mathrm{kg}$ lean body mass (LBM) between BC $(0.37 \pm 0.03 \mathrm{ng} \cdot \mathrm{mL}-1 \cdot \mathrm{kgLBM}-1 ; 95 \% \mathrm{Cl}$ : 0.28-0.47) and MBC $(2.89 \pm 0.24 \mathrm{ng} \cdot \mathrm{mL}-1 \cdot \mathrm{kgLBM}-1$; $95 \%$ Cl: 2.22-3.56).
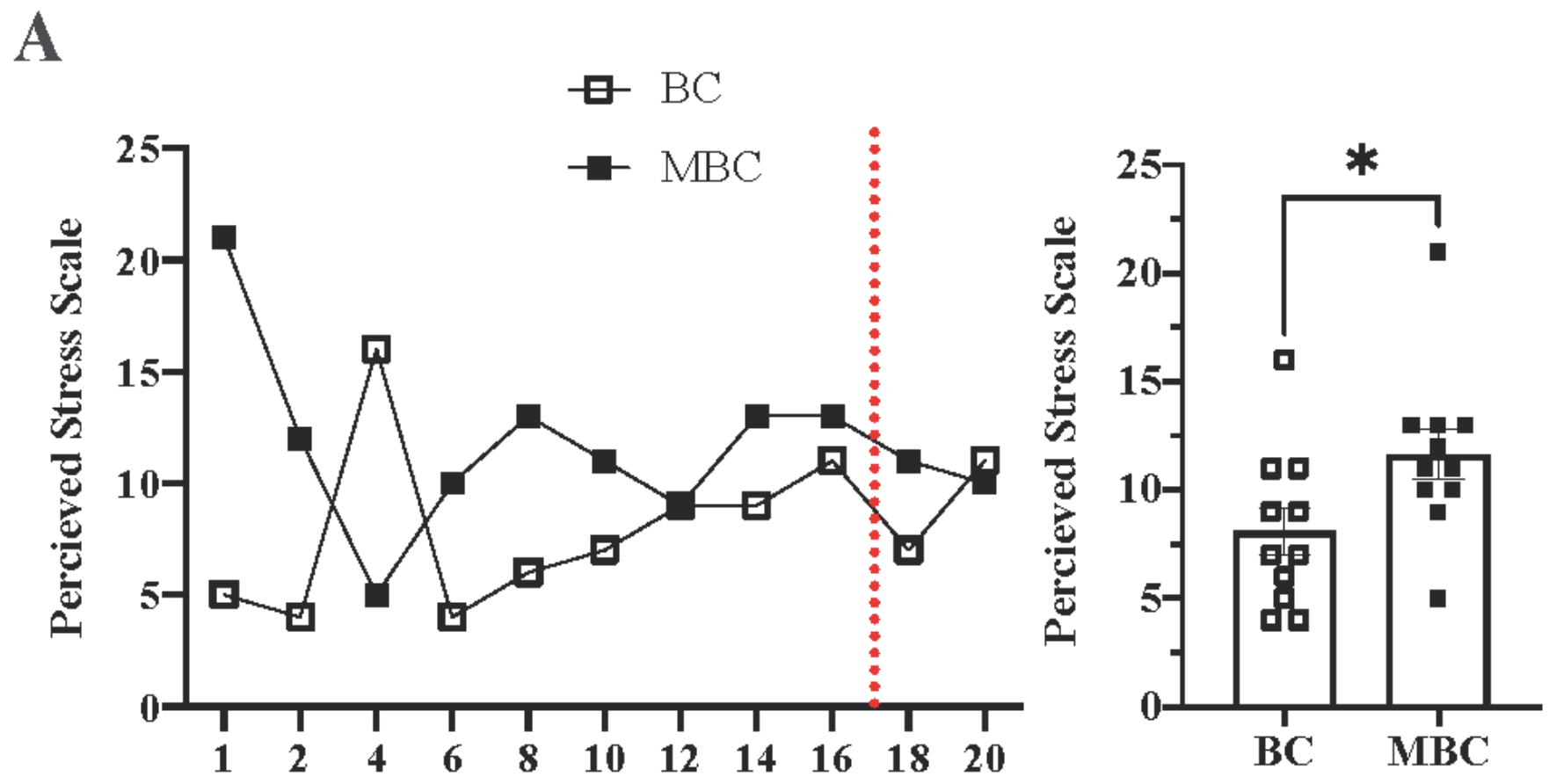

B
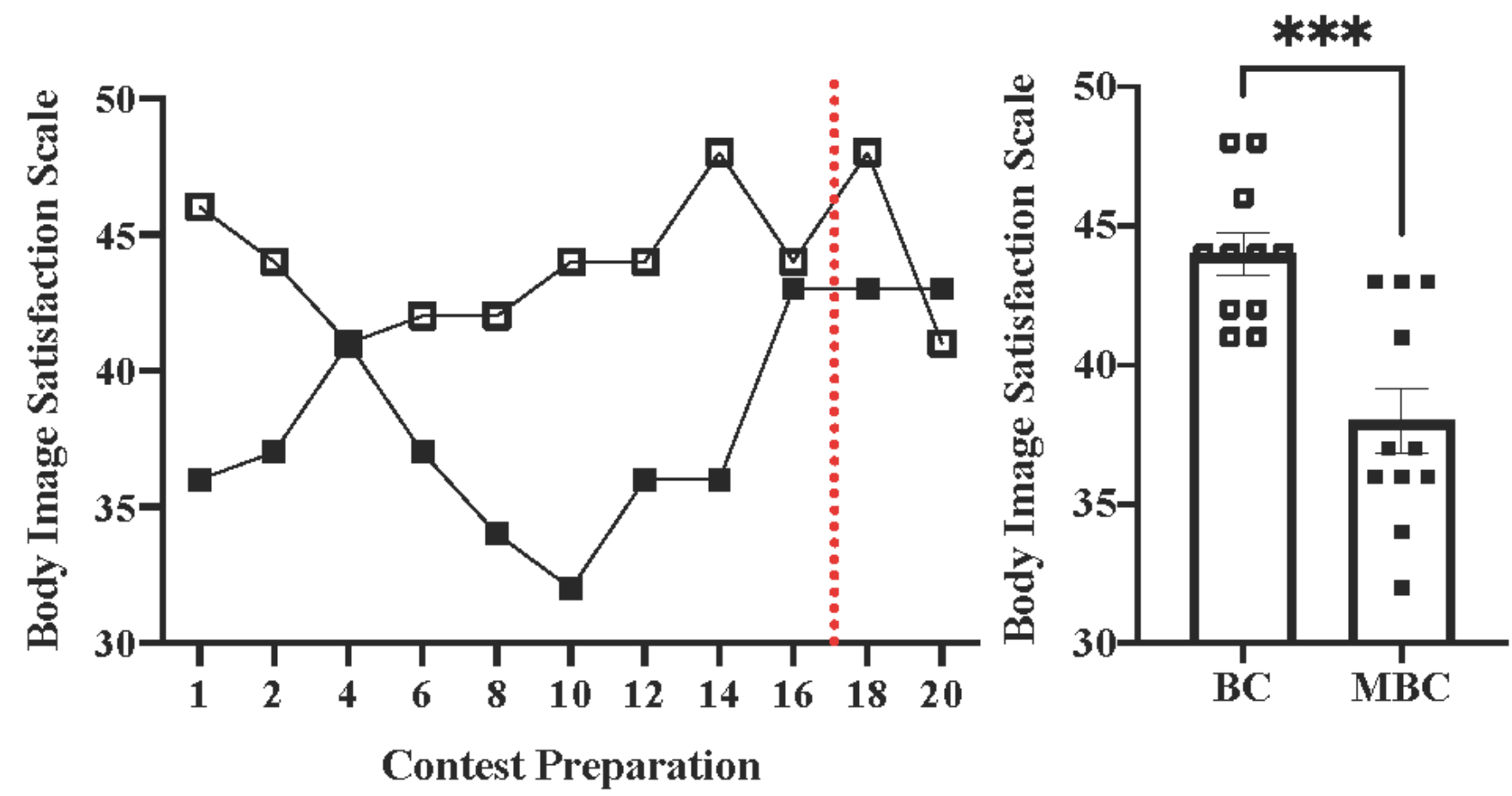

Figure 15 
The time course analysis of the impact of a 20-week contest preparation on body image and perceived stress with accompanying mean average (mean \pm SEM) comparison between $B C$ and MBC. A) A mean difference ( $p=0.03$ ) was found in the perceived stress scale (PSS) between BC $(8.09 \pm 1.09 ; 95 \% \mathrm{Cl}$ : 5.66-10.5) and MBC (11.64 $\pm 1.17 ; 95 \% \mathrm{Cl}$ : 9.03-14.24). B) A mean difference ( $\left.{ }^{* \star} p=0.0003\right)$ was found in the body image satisfaction scale (BISS) between BC (44.00 $\pm 0.75 ; 95 \% \mathrm{Cl}$ : 42.33-45.67) and MBC (38.00 $\pm 1.16 ; 95 \% \mathrm{Cl}: 35.40-40.60)$. The red dashed line denotes competition.

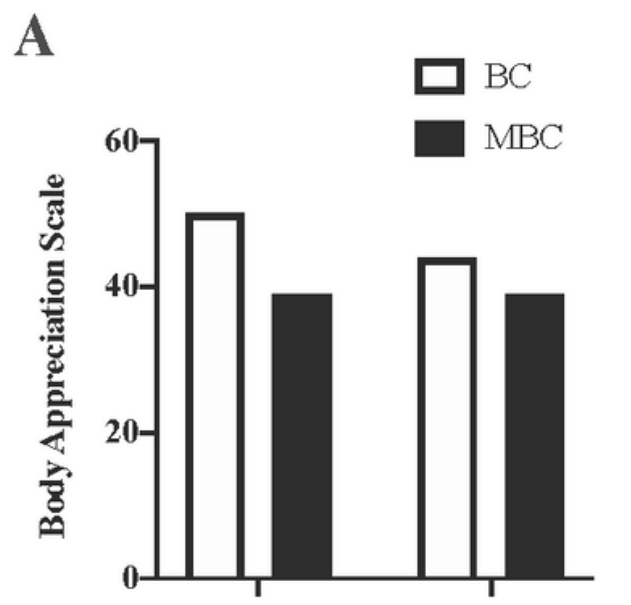

Baseline Post-Competition
B

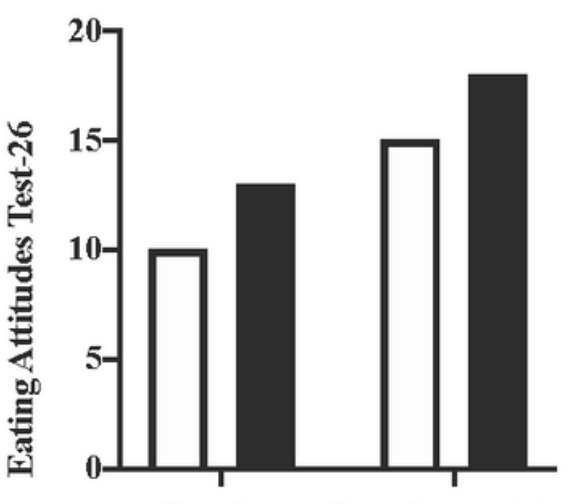

Baseline

Post-Competition
C

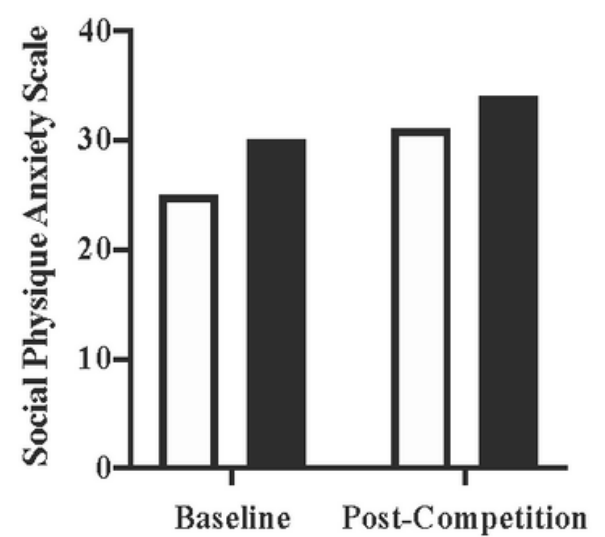

Figure 16

The psychometric analysis of the baseline and pre-competition on the A) body appreciation scale (BAS),

B) EAT-26, and C) social physique anxiety scale (SPAS) comparison between BC and MBC.

\section{Supplementary Files}

This is a list of supplementary files associated with this preprint. Click to download.

- Table4.pdf

- Table5.pdf

- Table6.pdf 